\title{
Zero-Energy Bound State Decay for Non-local Schrödinger Operators
}

\author{
Kamil Kaleta ${ }^{1}$, József Lớrinczi ${ }^{2}$ (i) \\ ${ }^{1}$ Faculty of Pure and Applied Mathematics, Wrocław University of Science and Technology, Wyb. \\ Wyspiańskiego 27, 50-370 Wrocław, Poland. E-mail: kamil.kaleta@pwr.edu.pl \\ 2 Department of Mathematical Sciences, Loughborough University, Loughborough LE11 3TU, UK. \\ E-mail: J.Lorinczi@lboro.ac.uk
}

Received: 18 March 2019 / Accepted: 28 May 2019

Published online: 17 July 2019 - (C) The Author(s) 2019

\begin{abstract}
We consider solutions of the eigenvalue equation at zero energy for a class of non-local Schrödinger operators with potentials decreasing to zero at infinity. Using a path integral approach, we obtain detailed results on the spatial decay at infinity of both $L^{2}$ and resonance solutions. We highlight the interplay of the kinetic term and the potential in these decay behaviours, and identify the decay mechanisms resulting from specific balances of global lifetimes with or without the potential.
\end{abstract}

\section{Introduction}

The study of spectral properties of Schrödinger operators $H=-\frac{1}{2} \Delta+V$ on $L^{2}\left(\mathbf{R}^{d}\right)$, featuring the Laplacian and a potential $V$, has a long history in mathematics. Potentials decaying to zero at infinity produce a fascinating variety of spectral behaviours and phenomenology, including the possibility of finite or countably infinite discrete spectra, (dense sets of) embedded eigenvalues or singular continuous spectrum, resonances, criticality, Efimov effect, enhanced binding, or scattering. In this, there is a split of qualitative behaviours according to the rates of decay of the potential (which led to concepts of 'long range' and 'short range' potentials). The results indicate that the existence of embedded eigenvalues is a long range effect, and the appearance of positive point spectrum is a combination of slow decay and oscillations of the potential. For surveys we refer to $[9,12,16,48]$ and the numerous references therein.

Zero-energy level, which coincides with the edge of the continuous spectrum, often marks a borderline between various regimes of spectral behaviour, in particular, between existence and non-existence of bound states, thus also shedding light on the mechanisms of "birth" of such states. Whether zero is an eigenvalue is, in general, a difficult problem. Some early results on existence or non-existence of zero-energy eigenvalues go back to

KK was supported by the National Science Center (Poland) Grant 2015/18/E/ST1/00239 and by the Alexander von Humboldt Foundation (Germany). JL thanks IHES, Bures-sur-Yvette, where part of this paper has been written. 
the papers $[1,25,30,32,33,35,44,46,47,56]$. For potentials which are negative at infinity, decaying at a rate $V(x) \asymp-c|x|^{-\gamma}, c>0$, as $|x| \rightarrow \infty$, and satisfying some further conditions, it has been established in [18] that for $\gamma \in(0,2)$ zero is not an eigenvalue, see also $[13,55]$. For potentials that are positive at infinity the situation changes $[43,59]$. In [4] it has been shown that for Schrödinger operators on $L^{2}\left(\mathbf{R}^{3}\right)$ with rotationally symmetric potentials $V \in L^{p}\left(\mathbf{R}^{3}\right), p>\frac{3}{2}$, whose positive part satisfies $V^{+}(x) \leq C|x|^{-2}$ for $x$ large enough, zero is not an eigenvalue corresponding to a positive eigenfunction if $C=\frac{3}{4}$, while a positive $L^{2}$-eigenfunction does exist if $C>\frac{3}{4}$. (The result holds more generally for non-symmetric potentials and higher dimensions as well.) For some further results on the existence of compactly supported eigenfunctions at zero-eigenvalue for compactly supported $V \in L^{p}\left(\mathbf{R}^{d}\right), p<\frac{d}{2}$, we refer to [31,34]. Apart from some cases using direct methods of analysis, the two most used techniques leading to these results are based on unique continuation or resolvent expansions.

Recently, a theory of non-local Schrödinger operators started to shape up, which has enriched the range of spectral phenomena and opened a new perspective to the understanding of classical Schrödinger operators as a specific case. Such operators $H=$ $L+V$ arise by replacing the Laplacian with a suitable pseudo-differential operator like the fractional Laplacian $L=(-\Delta)^{\alpha / 2}, 0<\alpha<2$. The $\alpha=1$ case is a model of a massless relativistic quantum particle in a potential $[15,38]$, while operators with other exponents have been used in the physics literature to describe photonic quantum effects $[36,61,62]$, laser cooling via trapping of particles [3], Lévy glasses [5], and other phenomena. For a further discussion of applications we refer to [28] and the references therein.

In the present paper our primary aim is an analysis of eigenfunction decay at zeroeigenvalue or zero-resonance for a class of non-local Schrödinger operators on $L^{2}\left(\mathbf{R}^{d}\right)$, with decaying potentials. One of our goals is to highlight the role of the potential in an interplay with the kinetic operator term $-L$ in generating the decay behaviours. Embedded eigenstates have been investigated in quantum theory since the seminal paper by von Neumann and Wigner [58], and led to applications in experimental physics, see e.g. $[7,8,42]$. We emphasize, however, that our focus in this paper is to eigenfunctions at the continuum threshold for a class of non-local operators, that is, we are not dealing with strictly positive eigenvalues and related eigenfunctions here. For emerging interest in this direction in the physics literature see $[14,60]$.

The occurrence of zero or strictly positive eigenvalues for non-local Schrödinger operators just begins to be mathematically studied. In the recent paper [39], see Theorems 2.8 and 2.10, two sets of potentials generating zero-eigenvalues or zero-resonances for massless relativistic Schrödinger operators in dimension one have been constructed (as well as examples leading to strictly positive eigenvalues). More recently, in [24] this has been extended to fractional Laplacians of all order and arbitrary dimensions. Let $\kappa>0, \alpha \in(0,2)$, and $P$ be a harmonic polynomial, homogeneous of degree $l \geq 0$, i.e., satisfying $P(c x)=c^{l} P(x)$ for all $c>0$, and $\Delta P=0$. Denote $\mu=d+2 l$, and consider the potentials and functions

$$
\begin{aligned}
V_{\kappa, \alpha}(x) & =-\frac{2^{\alpha}}{\Gamma(\kappa)} \Gamma\left(\frac{\mu+\alpha}{2}\right) \Gamma\left(\frac{\alpha}{2}+\kappa\right)\left(1+|x|^{2}\right)^{\kappa}{ }_{2} \mathbf{F}_{1}\left(\left.\frac{\mu+\alpha}{2} \frac{\mu^{\frac{\alpha}{2}}+\kappa}{2}|-| x\right|^{2}\right) \\
\varphi_{\kappa}(x) & =\frac{P(x)}{\left(1+|x|^{2}\right)^{\kappa}},
\end{aligned}
$$

where ${ }_{2} \mathbf{F}_{1}$ is Gauss' hypergeometric function. Then

$$
(-\Delta)^{\frac{\alpha}{2}} \varphi_{\kappa}+V_{\kappa, \alpha} \varphi_{\kappa}=0
$$


holds in distributional sense with $\varphi_{\kappa} \in L^{2}\left(\mathbf{R}^{d}\right)$ if $\kappa \geq \frac{\mu}{4}$, and

$$
\left|V_{\kappa, \alpha}(x)\right|= \begin{cases}O\left(|x|^{-\alpha}\right) & \text { if } \kappa \in\left(l, \frac{\mu}{2}\right) \backslash\left\{\frac{\mu-\alpha}{2}\right\} \\ O\left(|x|^{-2 \alpha}\right) & \text { if } \kappa=\frac{\mu-\alpha}{2} \\ O\left(|x|^{-\alpha} \log |x|\right) & \text { if } \kappa=\frac{\mu}{2} \\ O\left(|x|^{2 \kappa-\mu-\alpha}\right) & \text { if } \kappa \in\left(\frac{\mu}{2}, \frac{\mu+\alpha}{2}\right) .\end{cases}
$$

Furthermore, for large $|x|$ it follows that

$$
\begin{aligned}
& V_{\kappa, \alpha}(x)<0 \text { if } \quad \kappa \in\left(l, \frac{\mu-\alpha}{2}\right] \\
& V_{\kappa, \alpha}(x)>0 \text { if } \quad \kappa \in\left(\frac{\mu-\alpha}{2}, \frac{\mu+\alpha}{2}\right) .
\end{aligned}
$$

We note that the above examples by no means indicate that zero eigenvalues are common or easy to locate. By using methods of operator analysis, we have established further cases or conditions of existence as well as non-existence of embedded eigenvalues in [40]. For $d=3$ it is known that the operator $(-\Delta)^{1 / 2}+V$ has no non-negative eigenvalue provided that $|V|,|x \cdot \nabla V|$ and $|x \cdot \nabla(x \cdot \nabla V)|$ are jointly bounded by $C\left(1+x^{2}\right)^{-1 / 2}$, with a small $C>0$, see [49]. Related work on unique continuation for fractional Schrödinger equations imply further non-existence results $[17,50,53,54]$. Some further recent work include non-positive potentials with compact support and $L$ chosen to be the massive relativistic operator [41], and a class of generalized Schrödinger operators [10].

In our previous works [27,29] we have investigated eigenfunction decay for a large class of non-local Schrödinger operators. Using a path integration approach, $L$ was assumed to be the infinitesimal generator of a 'jump-paring' Lévy process, i.e., having the property that

$$
\int_{|x-y|>1,|y|>1} v(|x-y|) v(|y|) d y \leq C v(|x|), \quad|x|>1,
$$

where $v$ is the Lévy jump density entering the symbol of the kinetic part $-L$ of the operator (for details see the next section), and $C>0$ is a constant. This condition means that double (and by iteration, any multiple) large jumps are stochastically dominated by single large jumps, and it covers a large family of operators and related random processes of interest, including the fractional Laplacian and isotropic stable processes, relativistic Laplace operators and relativistic stable processes, etc. Classical Schrödinger operators can also be better understood against this backdrop as a limiting case, when the process becomes a Brownian motion and path continuity leads to singularly different behaviours.

Our approach based on path integration proved to be rather efficient, providing both sharp results and insight into the mechanisms governing the decay behaviours. In the first paper quoted above we considered confining potentials $V$ (increasing to infinity as $|x| \rightarrow \infty)$, and found that, for instance, if the potentials grow at a sufficiently regular rate in the sense that $\sup _{B} V \leq c \inf _{B} V$ for all unit balls $B$ far enough from the origin, with a constant $c>0$, then the ground state $\varphi_{0}$ behaves like $\varphi_{0} \asymp \frac{v}{V}$. In this case the contributions of the kinetic and potential terms in $H$ separate neatly. In the second paper we considered decaying potentials (decreasing to zero as $|x| \rightarrow \infty$ ), such that $\lambda_{0}=\inf \operatorname{Spec} H<0$. In this case both $V$ and $v$ decrease to zero, and now the interplay between the two terms determining the decay is more intricate. We have shown that the decay behaviour depends on how some quantities related to intrinsic jump preferences of 
the related Lévy processes compare with the gap between the ground state eigenvalue and the continuum edge. Specifically, if $\lambda_{0}$ is sufficiently low-lying, then $\varphi_{0} \asymp v$. However, when the gap is small and $v$ is chosen to have an increasingly light tail proceeding from a polynomial or sub-exponential, through an exponential, to a super-exponential decay rate, a sharp regime change can be observed and the rate of decay of $\varphi_{0}$ suddenly becomes slower than the rate of decay of $v$, see also [28].

The mechanisms generating these properties can be appreciated by the stochastic quantities entering the expressions. When $L$ is chosen such that the process $\left(X_{t}\right)_{t \geq 0}$ it generates has the jump-paring property and $V$ is an increasing potential, we obtain

$$
\varphi_{0}(x) \asymp \Lambda_{B(x, 1)} v(x), \text { with } \Lambda_{B(x, 1)}=\mathbf{E}^{x}\left[\int_{0}^{\tau_{B(x, 1)}} e^{-\int_{0}^{t} V\left(X_{s}\right) d s} d t\right],
$$

where $\tau_{B(x, 1)}=\inf \left\{t>0: X_{t} \notin B(x, 1)\right\}$ denotes the first exit time of the process from a unit ball centered in $x$, so that $\Lambda_{B(x, 1)}$ is the mean survival time in the given ball under the potential. This means that the fall-off rate depends on how soon the process perturbed by the potential on average leaves unit balls far out. Dependent on how negative $\lambda_{0}$ is and how light the tails of $v$ are, this relationship is preserved for decaying potentials up to a point when too light tails cause a drop below a critical level in the domination of single large jumps, which the ground state energy cannot compensate. Then the sojourn times due to multiple re-entries in unit balls of the process become comparable with the exit times and pile up 'backlogs' in the fall-off events, which slow the decay of $\varphi_{0}$ down. Indeed, when $v$ has so light tails that the jump-paring condition no longer holds, $\varphi_{0}$ decays necessarily (and possibly much) slower than $v$. In the extremal case of a classical Laplacian this will be reflected by the fact that while the transition probability density of Brownian motion (replacing the role of $v$ ) has Gaussian tails, ground states under decaying $V$ decrease only exponentially.

Our concern in the present paper is how $\varphi_{0}$ decays in the conditions when $\lambda_{0}=$ inf Spec $H=0$. We note that in classical results on ground state decay of usual (local) Schrödinger operators by Agmon, Carmona, and other authors (see a discussion, e.g., in $[37, \mathrm{Ch} .3]$ ), a gap between the lowest eigenvalue and the edge of the continuous spectrum is an essential ingredient, and the results break down when this gap is brought to zero. In [2] it has been shown that for $d \geq 2$ a zero-energy eigenfunction $\varphi$ for a potential $V$ satisfying $\left\||x|^{2-d / p} V\right\|_{L^{p}\left(\mathbf{R}^{d}\right)}<\infty$, for some $p \geq 1$ possibly being infinite, implies a power-law lower bound on the decay given by $|x|^{a} \varphi \notin L^{2}\left(\mathbf{R}^{d}\right)$, with some $a \in(0, \infty)$. The authors also showed that for a potential slower than $|x|^{-2}$, exponential decay of a zero-energy eigenfunction is possible. For some upper bounds on the decay rates see [21]. A more encompassing study of exponential decay has been made in [20], in which also decays faster than exponential have been ruled out.

Our framework for non-local Schrödinger operators with $\lambda_{0}<0$ discussed above does not extend to the zero eigenvalue case, however, we will develop here a new framework by using a restricted set of operators $L$, requiring a doubling property of the jump kernels instead of the more general jump-paring property. This includes fractional Schrödinger operators and others that are in a sense comparable. To the best of our knowledge, there were no attempts made so far in the literature using a similar path integration-based approach to understand embedded eigenstates at the continuum threshold. Our hope is that the techniques we propose here will contribute to a description of such states also for non-local Schrödinger operators having jump kernels with a lighter tail and, eventually, classical Schrödinger operators too, which will need further technical steps. 
In particular, from our approach it will be seen below that, since now $\lambda_{0}=0$, there is a complete lack of an energetic advantage from negative eigenvalues leading to the behaviours discussed above, and now the influence of the potential appears through vestigial effects resulting from its sign at infinity. This will also reflect in the fact that in this case the contributions of the exits and the re-entries in local neighbourhoods even out and add up to such an extent that (1.5) no longer holds, and the decay events become now governed by global lifetimes such as

$$
\Lambda_{B(x,|x| / 2)}=\mathbf{E}^{x}\left[\int_{0}^{\tau_{B(x,|x| / 2)}} e^{-\int_{0}^{t} V\left(X_{s}\right) d s} d t\right] .
$$

From this expression it can be appreciated that in the $\lambda_{0}=0$ case there is a rather delicate difference between the behaviour of paths under the perturbing potential and free fluctuations of the process, and this slight difference is responsible even for the very existence of a ground state at zero eigenvalue (for further details see Sect. 6).

Below we start from the assumption that, for a class of operators $L$ and decaying $V$, the eigenvalue equation $(-L+V) \varphi=0$ is satisfied by a function $\varphi \in L^{p}\left(\mathbf{R}^{d}\right)$, for some $p>0$, describing zero-energy eigenfunctions (when $p=2$ ) or zero-resonances (when $p \neq 2$ ). Then we will study the asymptotic behaviour of $\varphi(x)$ as $|x| \rightarrow \infty$ which, following from the choice of the input operators, has a pointwise decay to zero. Our main results for asymptotically positive potentials are Theorems 4.1-4.3, giving upper and lower bounds on $\varphi$ when these functions are positive or when they may have zeroes and distinct nodal domains. For asymptotically negative potentials we have Theorem 5.1, giving upper bounds. We note that our results apply to both zero-energy eigenfunctions and zero-resonances. As it will be seen in applications to specific cases (Theorems 6.16.4), these estimates perform remarkably well, giving exact or close hits of the precise asymptotics in (1.2) above.

The remainder of this paper is organized as follows. In Sect. 2 we introduce the class of non-local Schrödinger operators considered, and briefly summarize the relevant properties of the jump processes used in their Feynman-Kac representations. We also give an expression of the solutions of the eigenvalue equation using a path integral representation, which will be a key formula used throughout below. In Sect. 3 we derive and prove some self-improving upper and lower estimates on the solutions of related harmonic functions, on which our main conclusions will rely. In Sects. 4 and 5 we obtain the decay behaviours separately for potentials positive and negative at infinity, respectively. In the concluding Sect. 6 we illustrate these results on specific examples, and discuss some mechanisms lying behind these decays.

\section{Non-local Schrödinger Operators and Feynman-Kac Semigroups}

2.1. Non-local Schrödinger operators and related random processes. We start by a general remark on notation. A ball centered in $x \in \mathbf{R}^{d}$ and of radius $r>0$ will be denoted by $B(x, r)$, and its complement in $\mathbf{R}^{d}$ by $B(x, r)^{c}$. We write $a \wedge b=\min \{a, b\}$ and $a \vee b=\max \{a, b\}$. The notation $C(a, b, c, \ldots)$ will be used for a positive constant dependent on parameters $a, b, c, \ldots$, dependence on the operator $L$ or, equivalently, on the related Lévy process $\left(X_{t}\right)_{t \geq 0}$ will be indicated by $C(L)$ and $C(X)$, while dependence on the dimension $d$ is assumed without being stated explicitly. Since constants appearing in definitions and statements play a role, they will be numbered $C_{1}, C_{2}, \ldots$ so that they can be tracked. We will also use the notation $f \asymp C g$ meaning that $C^{-1} g \leq f \leq C g$ with a constant $C \geq 1$, while $f \asymp g$ means that there is a constant $C \geq 1$ such that 
the latter holds. By $f \approx g$ we understand that $\lim _{|x| \rightarrow \infty} f(x) / g(x)=1$. In proofs $c_{1}, c_{2}, \ldots$ will be used to denote auxiliary constants.

Consider the pseudo-differential operator $L$ with symbol $\psi$, defined by

$$
\widehat{L f}(\xi)=-\psi(\xi) \widehat{f}(\xi), \quad \xi \in \mathbf{R}^{d}, f \in \operatorname{Dom}(L),
$$

with dense domain $\operatorname{Dom}(L)=\left\{f \in L^{2}\left(\mathbf{R}^{d}\right): \psi \widehat{f} \in L^{2}\left(\mathbf{R}^{d}\right)\right\} \subset L^{2}\left(\mathbf{R}^{d}\right)$, and where the hats denote Fourier transform. We assume the symbol to be of the form

$$
\psi(\xi)=A \xi \cdot \xi+\int_{\mathbf{R}^{d} \backslash\{0\}}(1-\cos (\xi \cdot z)) v(d z),
$$

where $A=\left(a_{i j}\right)_{1 \leq i, j \leq d}$ is a symmetric non-negative definite matrix, and $v$ is a symmetric Radon measure on $\mathbf{R}^{\bar{d}} \backslash\{0\}$, i.e., $v(E)=v(-E)$, for every Borel set $E \subset \mathbf{R}^{d} \backslash\{0\}$, with the property that $\int_{\mathbf{R}^{d}}\left(1 \wedge|z|^{2}\right) v(d z)<\infty$. In the present paper we assume throughout without further notice that the measure $v(d z)$ has infinite total mass and it is absolutely continuous with respect to Lebesgue measure, i.e., $v\left(\mathbf{R}^{d} \backslash\{0\}\right)=\infty$ and $v(d z)=$ $v(z) d z$, with $v(z)>0, z \in \mathbf{R}^{d}$. For simplicity, we denote the density also by $v$. It is a standard fact [23] that $-L$ is a positive, self-adjoint operator with core $C_{0}^{\infty}\left(\mathbf{R}^{d}\right)$, and the expression

$$
L f(x)=\sum_{i, j=1}^{d} a_{i j} \frac{\partial^{2} f}{\partial x_{j} \partial x_{i}}(x)+\lim _{\varepsilon \downarrow 0} \int_{|z-x|>\varepsilon}(f(z)-f(x)) v(z-x) d z,
$$

for $x \in \mathbf{R}^{d}$ and $f \in C_{0}^{\infty}\left(\mathbf{R}^{d}\right)$ holds. Also, $\operatorname{Spec}(-L)=\operatorname{Spec}_{\mathrm{ess}}(-L)=[0, \infty)$.

Below we will use the symmetrization

$$
\Psi(r)=\sup _{|\xi| \leq r} \psi(\xi), \quad r>0,
$$

of the symbol $\psi$. Let $H(r)=\frac{\|A\|}{r^{2}}+\int_{\mathbf{R}^{d} \backslash\{0\}}\left(1 \wedge \frac{|z|^{2}}{r^{2}}\right) v(d z)$. A combination of [52, Rem. 4.8] and [45, Sect. 3] gives that

$$
C_{1} H\left(\frac{1}{r}\right) \leq \Psi(r) \leq C_{2} H\left(\frac{1}{r}\right), \quad r>0,
$$

holds with suitable constants $C_{1} \in(0,1), C_{2}>1$, independent of $A$ and $v$. Also, it follows directly that $r \mapsto H(r)$ is non-increasing and the doubling property $H(r) \leq$ $4 H(2 r), r>0$, holds, in particular, $\Psi(2 r) \leq 4\left(C_{2} / C_{1}\right) \Psi(r)$, for all $r>0$.

Furthermore, let

$$
\begin{gathered}
\mathcal{B}=\left\{f \in C_{\mathrm{c}}^{2}\left(\mathbf{R}^{d}\right): f(x)=1 \text { for } x \in B(0,1 / 2), f(x)=0\right. \\
\text { for } \left.x \in B(0,1)^{c} \text { and } 0 \leq f \leq 1\right\} .
\end{gathered}
$$

Then for $f_{s}(x)=f(x / s)$ with $f \in \mathcal{B}$ and $s>0$, the bounds

$$
\begin{aligned}
\left\|L f_{s}\right\|_{\infty} & \leq 2 v\left(B(0, s)^{c}\right)+\frac{1}{s^{2}} \sup _{i, j=1, \ldots, d}\left\|\frac{\partial^{2} f}{\partial x_{i} \partial x_{j}}\right\|_{\infty}\left(\|A\|+\int_{|y| \leq s}|y|^{2} v(d y)\right) \\
& \leq\left(2 \vee \sup _{i, j=1, \ldots, d}\left\|\frac{\partial^{2} f}{\partial x_{i} \partial x_{j}}\right\|_{\infty}\right) H(s), \quad s>0
\end{aligned}
$$


hold. Let $f_{s}(x)=f(x / s)$ for $s>0$, and denote

$$
C_{3}(L, s):=\inf _{f \in \mathcal{B}}\left\|L f_{s}\right\|_{\infty} .
$$

From the above observations it follows that

$$
C_{3}(L, s) \leq \frac{1}{C_{1}}\left(2 \vee \inf _{f \in \mathcal{B}} \sup _{i, j=1, \ldots, d}\left\|\frac{\partial^{2} f}{\partial x_{i} \partial x_{j}}\right\|_{\infty}\right) \Psi(1 / s), \quad s>0 .
$$

A special feature of the operators of the form (2.1) with (2.2) is that they can be treated by a path integral approach. For each choice of the matrix $A$ and the measure $v(d z)=v(z) d z$, the operator $L$ is the infinitesimal generator of an $\mathbf{R}^{d}$-valued rotationally symmetric Lévy process, $d \geq 1$, on the space of càdlàg paths (i.e., functions $[0, \infty) \rightarrow \mathbf{R}^{d}$ which are continuous from the right, having left limits). We denote by $\left(X_{t}\right)_{t \geq 0}$ the Lévy process generated by $L$, the probability measure of the process starting in $x \in \mathbf{R}^{d}$ by $\mathbf{P}^{x}$, and expectation with respect to $\mathbf{P}^{x}$ by $\mathbf{E}^{x}$. It is a general fact that $\left(X_{t}\right)_{t \geq 0}$ is a strong Markov process with respect to its natural filtration, and its characteristic function is given by

$$
\mathbf{E}^{0}\left[e^{i \xi \cdot X_{t}}\right]=e^{-t \psi(\xi)}, \quad \xi \in \mathbf{R}^{d}, t>0,
$$

where $\psi$ is the symbol of $-L$ as defined in (2.2) which, from a probabilistic perspective, is the Lévy-Khintchin formula for the class of Lévy processes we consider. In this context, $A$ is the diffusion matrix describing the Brownian component of the process $\left(X_{t}\right)_{t \geq 0}$, and $v(d z)$ is the jump measure (called Lévy measure) describing the jump component, while the drift is zero, thus the Lévy triplet of the process is $(0, A, v)$. When $A \equiv 0$, the random process $\left(X_{t}\right)_{t \geq 0}$ is a purely jump process, otherwise it contains an independent Brownian component.

The above properties jointly imply that $\left(X_{t}\right)_{t \geq 0}$ is a strong Feller process, or equivalently, its one-dimensional marginal distributions are absolutely continuous with respect to Lebesgue measure, i.e., there exist measurable functions $p(t, x, y)=p(t, 0, y-x)=$ : $p(t, y-x)$, corresponding to transition probability densities, such that $\mathbf{P}^{0}\left(X_{t} \in E\right)=$ $\int_{E} p(t, x) d x$, for every Borel set $E \subset \mathbf{R}^{d}$, see [51, Th. 27.7]. Let $D \subset \mathbf{R}^{d}$ be an open bounded set and consider the first exit time

$$
\tau_{D}=\inf \left\{t \geq 0: X_{t} \notin D\right\}
$$

from $D$. The transition probability densities $p_{D}(t, x, y)$ of the process killed on exiting $D$ are then given by the Dynkin-Hunt formula

$$
p_{D}(t, x, y)=p(t, y-x)-\mathbf{E}^{x}\left[p\left(t-\tau_{D}, y-X_{\tau_{D}}\right) ; \tau_{D}<t\right], \quad x, y \in D .
$$

The Green function of the process $\left(X_{t}\right)_{t \geq 0}$ on $D$ is thus $G_{D}(x, y)=\int_{0}^{\infty} p_{D}(t, x, y) d t$, for all $x, y \in D$.

We also recall that when $D \subset \mathbf{R}^{d}$ is a bounded open domain, the following formula due to Ikeda and Watanabe holds [22, Th. 1]: for every $\eta>0$ and every bounded or non-negative Borel function $f$ on $\mathbf{R}^{d}$ such that $\operatorname{dist}(\operatorname{supp} f, D)>0$, the equality

$$
\mathbf{E}^{x}\left[e^{-\eta \tau_{D}} f\left(X_{\tau_{D}}\right)\right]=\int_{D} \int_{0}^{\infty} e^{-\eta t} p_{D}(t, x, y) d t \int_{D^{c}} f(z) v(z-y) d z d y, \quad x \in D,
$$


holds. Furthermore, by [52, Rem. 4.8], the estimate

$$
\mathbf{E}^{0}\left[\tau_{B(0, r)}\right] \leq \frac{C_{4}}{\Psi(1 / r)}, \quad r>0
$$

follows with a constant $C_{4}$ independent of the process. For more details on Lévy processes and their generators we refer to $[23,51]$.

In the remainder of the paper we will use the following class of Lévy processes and related operators $L$.

Definition 2.1. Let $\left(X_{t}\right)_{t \geq 0}$ be a Lévy process with Lévy-Khintchin exponent $\psi$ as in (2.2) and Lévy triplet $(0, A, v)$, satisfying the following conditions.

(A1) There exist a non-increasing function $g:(0, \infty) \rightarrow(0, \infty)$ and constants $C_{5}$, $C_{6}>0$ such that

$$
v(x) \asymp C_{5} g(|x|), \quad x \in \mathbf{R}^{d} \backslash\{0\}, \quad \text { and } \quad g(|x|) \leq C_{6} g(2|x|), \quad|x| \geq 1 .
$$

(A2) There exists $t_{\mathrm{b}}>0$ such that $\sup _{x \in \mathbf{R}^{d}} p\left(t_{\mathrm{b}}, x\right)=p\left(t_{\mathrm{b}}, 0\right)<\infty$.

(A3) There exists a constant $C_{7}=C_{7}(X)$ such that

$$
\sup _{x, y:|x-y| \geq s / 8} G_{B(0, s)}(x, y) \leq C_{7} \frac{\Psi(1 / s)}{s^{d}}, \quad s \geq 1 .
$$

Assumption (A1) is a statement on the profile of $v$, including a doubling property. Assumption (A2) is equivalent with $e^{-t_{\mathrm{b}} \psi} \in L^{1}\left(\mathbf{R}^{d}\right)$, for some $t_{\mathrm{b}}>0$, which by the Markov property of $\left(X_{t}\right)_{t \geq 0}$ extends to every $t>t_{\mathrm{b}}$. Assumption (A3) is a technical condition on the Green function for particular balls. As it will be seen below, the class satisfying Assumptions (A1)-(A3) includes isotropic and anisotropic stable processes, i.e., fractional Schrödinger operators, and layered stable processes, corresponding to another class of Lévy-operators comparable with fractional Schrödinger operators, which will be discussed in Sect. 6.

Throughout this paper we will use X-Kato class potentials. We say that the Borel function $V: \mathbf{R}^{d} \rightarrow \mathbf{R}$, called potential, belongs to $\mathcal{K}^{X}$ associated with the Lévy process $\left(X_{t}\right)_{t \geq 0}$ if it satisfies

$$
\lim _{t \downarrow 0} \sup _{x \in \mathbf{R}^{d}} \mathbf{E}^{x}\left[\int_{0}^{t}\left|V\left(X_{S}\right)\right| d s\right]=0 .
$$

Also, we say that $V=V_{+}-V_{-}$is in $X$-Kato class whenever its positive and negative parts satisfy $V_{-} \in \mathcal{K}^{X}$ and $V_{+} \in \mathcal{K}_{\text {loc }}^{X}$, where $V_{+} \in \mathcal{K}_{\text {loc }}^{X}$ means that $V_{+} 1_{B} \in \mathcal{K}^{X}$ for all compact subsets $B \subset \mathbf{R}^{d}$. It is straightforward to see that $L_{\text {loc }}^{\infty}\left(\mathbf{R}^{d}\right) \subset \mathcal{K}_{\text {loc }}^{X}$, and by stochastic continuity of $\left(X_{t}\right)_{t \geq 0}$ also $\mathcal{K}_{\text {loc }}^{X} \subset L_{\text {loc }}^{1}\left(\mathbf{R}^{d}\right)$. Note that condition (2.10) allows local singularities of $V$.

With an operator $L$ given by (2.1) and an $X$-Kato class potential $V$, viewed as a multiplication operator, we call the operator

$$
H=-L+V \text { in } L^{2}\left(\mathbf{R}^{d}\right)
$$

defined by form-sum a non-local Schrödinger operator. To study the spectral properties of this operator, we use a Feynman-Kac type representation. 
2.2. Feynman-Kac semigroups and generalized eigenfunctions. Consider the oneparameter family of operators

$$
T_{t} f(x)=\mathbf{E}^{x}\left[e^{-\int_{0}^{t} V\left(X_{s}\right) d s} f\left(X_{t}\right)\right], \quad t>0 .
$$

By standard arguments based on Khasminskii's Lemma, see [37, Lem. 3.37-3.38], for an $X$-Kato class potential $V$ it follows that there exist constants $C_{8}(X, V), C_{9}(X, V)>0$ such that

$$
\sup _{x \in \mathbf{R}^{d}} \mathbf{E}^{x}\left[e^{-\int_{0}^{t} V\left(X_{s}\right) d s}\right] \leq \sup _{x \in \mathbf{R}^{d}} \mathbf{E}^{x}\left[e^{\int_{0}^{t} V_{-}\left(X_{s}\right) d s}\right] \leq C_{8} e^{C_{9} t}, \quad t>0 .
$$

This implies that the operators $T_{t}, t>0$, are well defined on every $L^{p}\left(\mathbf{R}^{d}\right), 1 \leq p \leq \infty$, and

$$
\left\|T_{t}\right\|_{p, p} \leq\left\|T_{t}\right\|_{\infty, \infty} \leq C_{8} e^{C_{9} t}, \quad t>0
$$

Moreover, the family $\left\{T_{t}: t \geq 0\right\}$ is a strongly continuous semigroup of operators on each $L^{p}\left(\mathbf{R}^{d}\right), 1 \leq p \leq \infty$, which we call the Feynman-Kac semigroup associated with the process $\left(X_{t}\right)_{t \geq 0}$ and potential $V$. We define

$$
H f=-\mathrm{s}-\lim _{t \downarrow 0} \frac{T_{t} f-f}{t}, \quad f \in L^{p}\left(\mathbf{R}^{d}\right),
$$

such that the limit exists in $L^{p}\left(\mathbf{R}^{d}\right)$. We denote the set of all such functions by $\operatorname{Dom}_{L^{p}} H$ and call it the $L^{p}$-domain on $H$. It is known that $H$ is a closed unbounded operator such that $\operatorname{Dom}_{L^{p}} H$ is dense in $L^{p}\left(\mathbf{R}^{d}\right)$. For $p=2$ the operator $H$ can be identified with a self-adjoint operator as given by (2.11), defined in a quadratic form sense [11, Ch. 2].

Next we summarize some basic properties of the operators $T_{t}$ which will be useful below. Recall that for a function $f \in L^{2}\left(\mathbf{R}^{d}\right)$ we write $f \geq 0$ (resp., $f>0$ ) if $f(x) \geq 0$ (resp., $f(x)>0$ ) almost everywhere in $\mathbf{R}^{d}$.

Lemma 2.1. Let $\left(X_{t}\right)_{t \geq 0}$ be a symmetric Lévy process with Lévy-Khintchin exponent satisfying (2.2) such that Assumption (A2) holds with some $t_{\mathrm{b}}>0$, and let $V$ be an $X$-Kato class potential. Then the following properties hold:

(1) For all non-negative Borel measurable functions $f, g$ the equality

$$
\int_{R^{d}} f(x) T_{t} g(x) d x=\int_{\mathbf{R}^{d}} T_{t} f(x) g(x) d x, \quad t>0,
$$

holds, i.e., $T_{t}$ are symmetric operators.

(2) The operators $T_{t}: L^{p}\left(\mathbf{R}^{d}\right) \rightarrow L^{\infty}\left(\mathbf{R}^{d}\right)$ for $1<p \leq \infty, t \geq t_{\mathrm{b}}$, and $T_{t}: L^{1}\left(\mathbf{R}^{d}\right) \rightarrow$ $L^{\infty}\left(\mathbf{R}^{d}\right)$ for $t \geq 2 t_{\mathrm{b}}$, are bounded.

(3) For all $t \geq 2 t_{\mathrm{b}}, T_{t}$ has a bounded measurable integral kernel $u(t, x, y)$, symmetric in $x$ and $y$, i.e., $T_{t} f(x)=\int_{\mathbf{R}^{d}} u(t, x, y) f(y) d y$, for all $f \in L^{p}\left(\mathbf{R}^{d}\right)$ and $1 \leq p \leq \infty$.

(4) For all $t>0$ and $f \in L^{\infty}\left(\mathbf{R}^{d}\right), T_{t} f$ is a bounded continuous function, that is $\left\{T_{t}: t \geq 0\right\}$ is a strongly Feller semigroup.

(5) For all $t>0$ the operators $T_{t}$ are positivity improving, i.e., $T_{t} f>0$ for all $f \in L^{2}\left(\mathbf{R}^{d}\right)$ such that $f \geq 0$ and $f \neq 0$ a.e. 
The proof of these general properties is left to the reader, which can be obtained as an extension to the present set-up of the facts in [37, Sects. 3.2-3.3]. Note that we do not assume that $p(t, x)$ is bounded for all $t>0$, and thus in general the operators $T_{t}: L^{p}\left(\mathbf{R}^{d}\right) \rightarrow L^{\infty}\left(\mathbf{R}^{d}\right)$ need not be bounded for $t<t_{\mathrm{b}}$.

Related to the Feynman-Kac semigroup, we define the potential operator by

$$
G^{V} f(x)=\int_{0}^{\infty} T_{t} f(x) d t=\mathbf{E}^{x}\left[\int_{0}^{\infty} e^{-\int_{0}^{t} V\left(X_{s}\right) d s} f\left(X_{t}\right) d t\right],
$$

for non-negative or bounded Borel functions $f$ on $\mathbf{R}^{d}$. Recall that $\tau_{D}$ denotes the first exit time of the process from domain $D$. Whenever $D \subset \mathbf{R}^{d}$ is an open set, it follows by the strong Markov property of the process that for every $x \in D$

$$
\begin{aligned}
& G^{V} f(x)=\mathbf{E}^{x}\left[\int_{0}^{\tau_{D}} e^{-\int_{0}^{t} V\left(X_{s}\right) d s} f\left(X_{t}\right) d t\right] \\
& +\mathbf{E}^{x}\left[e^{-\int_{0}^{\tau} D V\left(X_{s}\right) d s} G^{V} f\left(X_{\tau_{D}}\right) ; \tau_{D}<\infty\right] .
\end{aligned}
$$

For further information on potential theory we refer to [6].

Let $V$ be a decaying $X$-Kato class potential by which we mean $V(x) \rightarrow 0$ as $|x| \rightarrow$ $\infty$. The main object of our investigations in this paper are the solutions $\varphi \in L^{p}\left(\mathbf{R}^{d}\right)$, $p \geq 1, \varphi \not \equiv 0$, of the equation

$$
H \varphi=0,
$$

or, equivalently,

$$
T_{t} \varphi=\varphi, \quad t \geq 0
$$

In the $L^{2}$-framework

$$
\Sigma:=\inf \operatorname{Spec}_{\mathrm{ess}} H=\inf \operatorname{Spec}_{\mathrm{ess}}(-L)=0
$$

is the edge of the essential spectrum of $H$ and (2.14) can be understood as the eigenvalue equation at $\Sigma$. Whenever the solution $\varphi$ to (2.14) is such that $\varphi \in \operatorname{Dom}_{L^{2}} H$, we call it a zero-energy eigenfunction (or zero-energy bound state) and then 0 is an eigenvalue. Otherwise, we call both (by a slight abuse of language) a zero-resonance.

Throughout we will assume that every zero-energy eigenfunction $\varphi$ is $L^{2}$-normalized so that $\|\varphi\|_{2}=1$. Moreover, by Lemma 2.1 it follows that $T_{t}\left(L^{p}\left(\mathbf{R}^{d}\right)\right) \subset L^{\infty}\left(\mathbf{R}^{d}\right)$ and $T_{t}\left(L^{\infty}\left(\mathbf{R}^{d}\right)\right) \subset C_{\mathrm{b}}\left(\mathbf{R}^{d}\right)$ for every $t>2 t_{\mathrm{b}}$ and $p \geq 1$. Therefore, any solution $\varphi$ to (2.14) and (2.15) is a bounded and continuous function, in particular, it makes sense to study their pointwise estimates.

Below we will make frequently use of the following resolvent representation of solutions of (2.14). Choose $\theta>0$. Then by multiplying both sides of (2.15) and integrating with respect to time, we obtain

$$
\varphi(x)=\theta(\theta+H)^{-1} \varphi=\theta \int_{0}^{\infty} \mathbf{E}^{x}\left[e^{-\int_{0}^{t}\left(\theta+V\left(X_{s}\right)\right) d s} \varphi\left(X_{t}\right)\right] d t, \quad x \in \mathbf{R}^{d} .
$$


Combining this with (2.13) applied to $f=\varphi$ for an arbitrary open set $D \subset \mathbf{R}^{d}$ and $x \in D$, and using the strong Markov property of the Lévy process $\left(X_{t}\right)_{t \geq 0}$, we readily obtain

$$
\begin{aligned}
\varphi(x)= & \theta\left(\int_{0}^{\tau_{D}}+\int_{\tau_{D}}^{\infty}\right) \mathbf{E}^{x}\left[e^{-\int_{0}^{t}\left(\theta+V\left(X_{s}\right)\right) d s} \varphi\left(X_{t}\right)\right] d t \\
= & \theta \mathbf{E}^{x}\left[\int_{0}^{\tau_{D}} e^{-\int_{0}^{t}\left(\theta+V\left(X_{s}\right)\right) d s} \varphi\left(X_{t}\right) d t\right] \\
& +\mathbf{E}^{x}\left[e^{-\int_{0}^{\tau_{D}}\left(\theta+V\left(X_{s}\right)\right) d s} \varphi\left(X_{\tau_{D}}\right) ; \tau_{D}<\infty\right],
\end{aligned}
$$

which will be a fundamental formula in what follows.

\section{Self-improving Estimates}

In this section we show some key estimates which will serve to proving our main results below. Let $R_{0} \geq 1$ be a fixed number. Throughout this section we will consider nonincreasing functions $u, v, w:\left[R_{0}, \infty\right) \rightarrow(0, \infty)$ such that there exist $C_{10}, C_{12} \geq 1$ satisfying

$$
u(r) \leq C_{10} u(2 r) \quad w(r) \leq C_{12} w(2 r), \quad r \geq R_{0}
$$

and

$$
\int_{|x|>r} u(|x|) d x \leq w(r), \quad r \geq R_{0} .
$$

In what follows we will also use the notations

$$
K_{u, v}:=\frac{u}{v} \quad \text { and } \quad h_{u, v}(r):=\int_{R_{0} \leq|y| \leq r} K_{u, v}(|y|) d y, \quad r \geq R_{0},
$$

and $\omega_{d}$ will denote the volume of a unit $d$-dimensional ball.

Lemma 3.1. Let $u, v, w:\left[R_{0}, \infty\right) \rightarrow(0, \infty)$ be non-increasing functions such that (3.1) and (3.2) hold with constants $C_{10}, C_{12} \geq 1$ and

$$
\lim _{r \rightarrow \infty} \frac{w(r)}{v(r)}=0 \text {. }
$$

Moreover, suppose that $f$ is a bounded non-negative function on $\mathbf{R}^{d}$ such that

$$
f(x) \leq \frac{C_{13}}{v(|x|)}\left(\int_{|z-x|>\frac{|x|}{2}} f(z) u(|z-x|) d z+\frac{w(|x|)}{|x|^{d}} \int_{\frac{|x|}{32}<|z-x| \leq \frac{|x|}{2}} f(z) d z\right),
$$

for $|x| \geq R_{0}$ and a constant $C_{13}>0$, and let $\eta:=C_{10} C_{13}$. If

$$
\sup _{r>R_{0}} K_{u, v}(r) e^{\eta h_{u, v}(r)}<\infty
$$

then there exist $R>2 R_{0}$ and $C_{14}=C_{14}(R)$ such that

$$
f(x) \leq C_{14}\|f\|_{\infty} K_{u, v}(|x|) e^{\eta h_{u, v}(|x|)}, \quad|x| \geq R .
$$


In particular, if

$$
\int_{|y| \geq R_{0}} K_{u, v}(|y|) d y<\infty
$$

then

$$
f(x) \leq C_{14} e^{\eta h_{u, v}(\infty)}\|f\|_{\infty} K_{u, v}(|x|), \quad|x| \geq R, \quad \text { with } h_{u, v}(\infty):=\lim _{r \rightarrow \infty} h_{u, v}(r) .
$$

Proof. Observe that (3.8) follows directly from (3.6). We only need to prove (3.6). Let

$$
c_{1}:=\sup _{r \geq R_{0}} K_{u, v}(r) e^{\eta h_{u, v}(r)}
$$

and $R>2 R_{0}$ be large enough such that

$$
c_{2}:=\sup _{|x| \geq R} \frac{w(|x|)}{v(|x|)}<\frac{1}{\left(1+c_{1}\right) C_{13}\left(C_{12}+2^{-d}\right)} .
$$

By (3.4) and the part of (3.1) stated for $u$, for every $|x| \geq R$ we obtain

$$
\begin{aligned}
& f(x) \leq \frac{C_{13}}{v(|x|)}\left(\int_{\substack{|z-x|>\frac{|x|}{|z| \leq R} \\
\mid}} f(z) u(|z-x|) d z+\frac{w(|x|) \mathbf{1}_{\{|x| \leq 2 R\}}}{|x|^{d}} \int_{\frac{|x|}{32}<|z-x| \leq \frac{|x|}{2}} f(z) d z\right) \\
& +\frac{C_{13}}{v(|x|)}\left(\int_{\substack{|z-x|>\frac{|x|}{2} \\
|z|>R}} f(z) u(|z-x|) d z+\frac{w(|x|) \mathbf{1}_{\{|x|>2 R\}}}{|x|^{d}} \int_{\frac{|x|}{32}<|z-x| \leq \frac{|x|}{2}} f(z) d z\right) \\
& \leq \frac{C_{13}\|f\|_{\infty}}{v(|x|)}\left(\omega_{d} R^{d} u(|x| / 2)+\frac{\omega_{d} w(2 R)}{2^{d} u(2 R)} u(|x|)\right) \\
& +\frac{C_{13}}{v(|x|)}\left(\int_{\substack{|z-x|>\frac{|x|}{\mid}|z|>R \\
\mid}} f(z) u(|z-x|) d z+\frac{w(|x|) \mathbf{1}_{\{|x|>2 R\}}}{|x|^{d}} \int_{\frac{|x|}{32}<|z-x| \leq \frac{|x|}{2}} f(z) d z\right) \\
& \leq C_{13} \omega_{d}\left(C_{10} R^{d}+\frac{w(2 R)}{2^{d} u(2 R)}\right)\|f\|_{\infty} \frac{u(|x|)}{v(|x|)} \\
& +\frac{C_{13}}{v(|x|)}\left(\int_{\substack{|z-x|>\frac{|x|}{2}|z|>R \\
\mid}} f(z) u(|z-x|) d z+\frac{w(|x|)}{|x|^{d}} \int_{\substack{|x| \\
32<|z-x| \leq \frac{|x|}{2}|z|>R}} f(z) d z\right) .
\end{aligned}
$$

From this we obtain the two independent estimates

$$
\begin{aligned}
f(x) \leq & C_{13} \omega_{d}\left(C_{10} R^{d}+\frac{w(2 R)}{2^{d} u(2 R)}\right)\|f\|_{\infty} \frac{u(|x|)}{v(|x|)} \\
& +\frac{C_{13}}{v(|x|)} \int_{\substack{R \leq|z| \leq|x| \\
|x-z|>\frac{|x|}{2}}} f(z) u(|x-z|) d z \\
& +C_{13} \frac{w(|x|)}{v(|x|)}\left(\frac{\int_{|z|>\frac{|x|}{2}} u(|z|) d z}{w(|x|)}+\frac{1}{2^{d}}\right) \sup _{\substack{|z| \geq \frac{|x|}{2} \vee R \\
\mid z}} f(z)
\end{aligned}
$$


and

$$
\begin{aligned}
f(x) \leq & C_{13} \omega_{d}\left(C_{10} R^{d}+\frac{w(2 R)}{2^{d} u(2 R)}\right)\|f\|_{\infty} \frac{u(|x|)}{v(|x|)} \\
& +C_{13}\|f\|_{\infty} \frac{w(|x|)}{v(|x|)}\left(\frac{\int_{|z|>\frac{|x|}{2}} u(|z|) d z}{w(|x|)}+\frac{1}{2^{d}}\right) .
\end{aligned}
$$

They give, respectively,

$$
\begin{aligned}
f(x) \leq & c_{3}\|f\|_{\infty} \frac{u(|x|)}{v(|x|)}+\frac{C_{13}}{v(|x|)} \int_{\substack{R \leq|z| \leq|x| \\
|x-z|>\frac{|x|}{2}}} f(z) u(|x-z|) d z \\
& +c_{2} C_{13}\left(C_{12}+\frac{1}{2^{d}}\right) \sup _{\substack{|z| \geq \frac{|x|}{2} \vee R\\
}(z)} f(z)
\end{aligned}
$$

and

$$
f(x) \leq c_{3}\|f\|_{\infty}\left(\frac{u(|x|)}{v(|x|)}+c_{2} C_{13}\left(C_{12}+2^{-d}\right)\right),
$$

for $|x| \geq R$, where

$$
c_{3}:=C_{13} \omega_{d}\left(C_{10} R^{d}+\frac{w(2 R)}{2^{d} u(2 R)}\right) \vee 1 .
$$

Also, recall that $\eta=C_{10} C_{13}$, denote $c_{4}=c_{2}\left(1+c_{1}\right) C_{13}\left(C_{12}+2^{-d}\right)$, and notice that by (3.9) and (3.10) it follows that $c_{4}<1$.

Now we show that for every $p \in \mathbf{N}$

$$
f(x) \leq c_{3}\|f\|_{\infty}\left[K_{u, v}(|x|) \sum_{k=1}^{p} \frac{\left(\eta h_{u, v}(|x|)\right)^{k-1}}{(k-1) !}+c_{4}^{p}\right], \quad|x| \geq R .
$$

Notice that if this holds, then by taking the limit $p \rightarrow \infty$ it follows that

$$
f(x) \leq c_{3}\|f\|_{\infty} K_{u, v}(|x|) e^{\eta h_{u, v}(|x|)}, \quad|x| \geq R,
$$

which is the bound stated in the lemma. To prove (3.13) we perform induction on $p \in \mathbf{N}$. First observe that for $p=1$ the estimate (3.13) follows from (3.12). Suppose now that (3.13) holds for $p-1 \in \mathbf{N}$. By using (3.11) and the induction hypothesis, we see for all $|x| \geq R$ that

$$
\begin{aligned}
f(x) \leq & c_{3}\|f\|_{\infty} K_{u, v}(|x|) \\
& +c_{3} C_{10} C_{13}\|f\|_{\infty} K_{u, v}(|x|) \sum_{k=1}^{p-1} \frac{\eta^{k-1}}{(k-1) !} \int_{1 \leq|z| \leq|x|} K_{u, v}(|z|) h_{u, v}(|z|)^{k-1} d z \\
& +c_{3}\|f\|_{\infty}\left(c_{2} C_{12} C_{13}+c_{1} c_{2} C_{13}\left(C_{12}+2^{-d}\right)+c_{2} C_{13}\left(C_{12}+2^{-d}\right)\right) c_{4}^{p-1} .
\end{aligned}
$$


By a substitution we obtain

$$
\begin{aligned}
& \int_{R_{0} \leq|z| \leq|x|} K_{u, v}(|z|) h_{u, v}(|z|)^{k-1} d z \\
& \quad=\int_{R_{0} \leq\left|z_{2}\right| \leq|x|} K_{u, v}\left(\left|z_{2}\right|\right)\left(\int_{R_{0} \leq\left|z_{1}\right| \leq\left|z_{2}\right|} K_{u, v}\left(\left|z_{1}\right|\right) d z_{1}\right)^{k-1} d z_{2} \\
& =\omega_{d-1}^{k} \int_{R_{0}}^{|x|} K_{u, v}\left(\rho_{2}\right) \rho_{2}^{d-1}\left(\int_{R_{0}}^{\rho_{2}} K_{u, v}\left(\rho_{1}\right) \rho_{1}^{d-1} d \rho_{1}\right)^{k-1} d \rho_{2} \\
& =\frac{\omega_{d-1}^{k}}{k}\left(\int_{R_{0}}^{|x|} K_{u, v}\left(\rho_{1}\right) \rho_{1}^{d-1} d \rho_{1}\right)^{k} \\
& =\frac{1}{k}\left(\int_{R_{0} \leq|z| \leq|x|} K_{u, v}(|z|) d z\right)^{k}
\end{aligned}
$$

and thus

$$
\begin{aligned}
f(x) & \leq c_{3}\|f\|_{\infty} K_{u, v}(|x|)+c_{3}\|f\|_{\infty} K_{u, v}(|x|) \sum_{k=1}^{p-1} \frac{\eta^{k}}{k !} h_{u, v}(|x|)^{k}+c_{3}\|f\|_{\infty} c_{4}^{p} \\
& =c_{3}\|f\|_{\infty}\left[K_{u, v}(|x|) \sum_{k=1}^{p} \frac{\left(\eta h_{u, v}(|x|)\right)^{k-1}}{(k-1) !}+c_{4}^{p}\right], \quad|x| \geq R,
\end{aligned}
$$

which is the claimed bound.

Remark 3.1. With some extra work it is possible to check that under the non-restrictive assumption that the function $K_{u, v}$ is almost non-increasing, i.e., there exists $C \geq 1$ such that $K_{u, v}(s) \leq C K_{u, v}(r)$ for all $R_{0} \leq r \leq s$, condition (3.7) can be understood also in terms of a convolution condition. Indeed, if (3.7) holds, then

$$
\sup _{|x| \geq 2 R_{0}} \frac{\int_{|z-x|>R_{0},|z|>R_{0}} K_{u, v}(|x-z|) K_{u, v}(|z|) d z}{K_{u, v}(|x| / 2)}<\infty,
$$

and a converse property is that

$$
\sup _{|x| \geq R_{0}} \frac{\int_{|z-x|>R_{0},|z|>R_{0}} K_{u, v}(|x-z|) K_{u, v}(|z|) d z}{K_{u, v}(|x|)}<\infty
$$

implies (3.7). The proof involves some lengthy but simple calculations, and the details are left to the interested reader.

The next lemma deals with a lower bound on positive functions satisfying an integral inequality.

Lemma 3.2. Let $u, v:[1, \infty) \rightarrow(0, \infty)$ be non-increasing functions such that $u$ satisfies (3.1) with a constant $C_{10} \geq 1$, and suppose that $f$ is a positive function on $\mathbf{R}^{d}$ such that

$$
f(x) \geq \frac{C_{15}}{v(|x|)} \int_{\substack{|z|<|x| \\|z+x|<|z-x|}} f(z) u(|z-x|) d z, \quad|x|>1,
$$


for a constant $C_{15}>0$. Then

$$
f(x) \geq \eta e^{-\eta h_{u, v}(1)} K_{u, v}(|x|) e^{\eta h_{u, v}(|x|)}, \quad|x|>1,
$$

with constant

$$
\eta:=\frac{C_{15}}{C_{10}}\left(\frac{1}{2} \wedge \inf _{x:|x|=1} \int_{\substack{|z| \leq 1 \\|z+x|<|z-x|}} f(z) d z\right)
$$

In particular,

$$
f(x) \geq \eta K_{u, v}(|x|), \quad|x|>1 .
$$

Proof. By (3.15) we get

$$
f(x) \geq \frac{C_{15}}{C_{10}} \frac{u(|x|)}{v(|x|)}\left(\int_{\substack{|z| \leq 1 \\|z+x|<|z-x|}} f(z) d z+\int_{\substack{1<|z|<|x| \\|z+x|<|z-x|}} f(z) d z\right), \quad|x|>1,
$$

and by symmetrization of the second integral,

$$
\begin{aligned}
f(x) & \geq \frac{C_{15}}{C_{10}} K_{u, v}(|x|)\left(\int_{\substack{|z| \leq 1 \\
|z+x|<|z-x|}} f(z) d z\right. \\
& \left.+\frac{1}{2}\left(\int_{\substack{1<|z|<|x| \\
|z+x|<|z-x|}} f(z) d z+\int_{\substack{1<|z|<|x| \\
|z-x|<|z+x|}} f(-z) d z\right)\right),
\end{aligned}
$$

for all $|x|>1$. Next we prove that for every $p \in \mathbf{N}$

$$
f(x) \geq \eta K_{u, v}(|x|) \sum_{k=1}^{p} \frac{\left(\eta\left(h_{u, v}(|x|)-h_{u, v}(1)\right)\right)^{k-1}}{(k-1) !}, \quad|x|>1,
$$

holds with

$$
\eta:=\frac{C_{15}}{C_{10}}\left(\frac{1}{2} \wedge \inf _{|x|=1} \int_{\substack{|z| \leq 1 \\|z+x|<|z-x|}} f(z) d z\right) .
$$

Clearly, if (3.19) is true for every $p \in \mathbf{N}$, then estimate (3.16) also holds.

We use induction on $p$. For $p=1$ the inequality (3.19) is an immediate consequence of (3.18). Suppose now that the induction hypothesis holds for some $p \in \mathbf{N}$. By (3.18) and (3.19) and rotation symmetry we obtain

$$
f(x) \geq \eta K_{u, v}(|x|)\left(1+\eta \sum_{k=1}^{p} \frac{\eta^{k-1}}{(k-1) !} \int_{1<|z|<|x|} \frac{u(|z|)}{v(|z|)}\left(h_{u, v}(|z|)-h_{u, v}(1)\right)^{k-1} d z\right),
$$

for $|x|>1$. Similarly as in the calculation above leading to (3.14), we obtain

$$
\begin{aligned}
& \int_{1<|z|<|x|} \frac{u(|z|)}{v(|z|)}\left(h_{u, v}(|z|)-h_{u, v}(1)\right)^{k-1} d z \\
& =\omega_{d-1} \int_{1}^{|x|} \frac{u(r)}{v(r)}\left(h_{u, v}(r)-h_{u, v}(1)\right)^{k-1} r^{d-1} d r \\
& =\frac{\left(h_{u, v}(|x|)-h_{u, v}(1)\right)^{k}}{k}, \quad|x|>1,
\end{aligned}
$$


and thus we conclude that

$$
\begin{aligned}
f(x) & \geq \eta K_{u, v}(|x|)\left(1+\sum_{k=1}^{p} \frac{\eta^{k}}{k !}\left(h_{u, v}(|x|)-h_{u, v}(1)\right)^{k}\right) \\
& =\eta K_{u, v}(|x|) \sum_{k=1}^{p+1} \frac{\eta^{k-1}}{(k-1) !}\left(h_{u, v}(|x|)-h_{u, v}(1)\right)^{k-1}, \quad|x|>1,
\end{aligned}
$$

as required.

\section{Decay of Zero-Energy Eigenfunctions for Potentials Positive at Infinity}

4.1. Upper bound. Now we turn to discussing the spatial decay properties of eigenfunctions of non-local Schrödinger operators presented in Sect. 2. In this section we consider decaying potentials that are non-negative at infinity in the following sense:

(A4) $V$ is an $X$-Kato class potential such that $V(x) \rightarrow 0$ as $|x| \rightarrow \infty$, and there exists $r_{0}>0$ such that $V(x) \geq 0$ for $|x| \geq r_{0}$.

It will be useful to introduce the notation

$$
V_{*}(x):=\inf _{r_{0} \leq|y| \leq \frac{3}{2}|x|} V(y), \quad|x| \geq r_{0} .
$$

Notice that $V_{*}(x)$ is a radial and non-increasing function such that $V_{*}(x) \geq 0,|x| \geq r_{0}$.

We will need a uniform estimate of functions that are harmonic with respect to the operator $H$. Since our approach is via a Feynman-Kac type stochastic representation, we use throughout the following probabilistic definition. Let $D$ be an open subset of $\mathbf{R}^{d}$ and let $V$ be a Kato-class potential such that $V(x) \geq 0$ on $D$. We call a non-negative Borel function $f$ on $\mathbf{R}^{d}$ an $(X, V)$-harmonic function in the domain $D$ if

$$
f(x)=\mathbf{E}^{x}\left[e^{-\int_{0}^{\tau_{U}} V\left(X_{s}\right) d s} f\left(X_{\tau_{U}}\right) ; \tau_{U}<\infty\right], \quad x \in U,
$$

for every open set $U$ with its closure $\bar{U}$ contained in $D$, and a regular $(X, V)$-harmonic function in $D$ if (4.1) holds for $U=D$ (where $\tau_{U}$ is the first exit time from $U$ ). By the strong Markov property every regular $(X, V)$-harmonic function in $D$ is $(X, V)$ harmonic in $D$. Whenever $V \equiv 0$ in $D$, we refer to $f$ as a (regular) $X$-harmonic function.

An initial version of the type of bound we prove below has been first obtained in [29, Lem. 3.1] and it can be derived from the general results in [6]. Here we need a variant suitable for the purposes of the present paper; note that the following estimate does not exclude the case $V \equiv 0$. Recall the expression (2.3).

Lemma 4.1. Let $\left(X_{t}\right)_{t \geq 0}$ be a Lévy process with Lévy-Khintchin exponent $\psi$ as in (2.2) such that Assumptions (A1)-(A3) and (A4) hold; specifically, let (A4) hold with some $r_{0}>0$. Then for every $\eta \in\left(0, \frac{1}{4}\right]$ there exists a constant $C_{16}>0$ such that for every non-negative function $f$ on $\mathbf{R}^{d}$ which is regular $(X, V)$-harmonic in a ball $B(x, \eta|x|)$, $|x| \geq r_{0} /(1-\eta)$, the bound

$$
f(y) \leq \frac{C_{16}}{V_{*}(x) \vee \Psi\left(\frac{1}{|x|}\right)}\left(\int_{|z-x|>2 \eta|x|} f(z) v(z-x) d z+\frac{\Psi\left(\frac{1}{|x|}\right)}{|x|^{d}} \int_{\frac{\eta|x|}{8}<|x-z| \leq 2 \eta|x|} f(z) d z\right),
$$


is satisfied, whenever $|x-y|<\frac{\eta|x|}{32}$.

Proof. We borrow some notation from [29, Sect. 3.2]. Let $0<s_{1}<s_{2}<s_{3} \leq \infty$ and define

$$
K_{2}\left(s_{1}, s_{2}, s_{3}\right):=\inf \left\{C \geq 1: v(x-y) \leq C v(x),|y| \leq s_{1}, s_{2} \leq|x|<s_{3}\right\} .
$$

Using (A1) of Definition 2.1, it is seen that $K_{2}\left(s_{1}, s_{2}, s_{3}\right)$ is well-defined and a nondecreasing function in $s_{1} \in\left(0, s_{2}\right)$, for every fixed $0<s_{2}<s_{3} \leq \infty$. Also, using (A3) of the same definition, we define

$$
K_{3}(s):=\sup _{x, y:|x-y| \geq s / 8} G_{B(0, s)}(x, y), \quad s>0 .
$$

For a detailed explanation of the role and probabilistic interpretation of these parameter functions we refer the reader to the discussion of Eqs. (2.13) and (2.14) in [29]. Then, for $s_{1} \geq 1$ and $s_{2}=2 s_{1}$, define

$$
h_{1}\left(s_{1}, s_{2}\right)=K_{2}\left(s_{1}, s_{2}, \infty\right)\left[C_{3}\left(L, \frac{s_{1}}{16}\right)\left(C_{17}\left(s_{1}\right)\left|B\left(0, s_{1}\right)\right|+\mathbf{E}^{0}\left[\tau_{B\left(0,2 s_{1}\right)}\right]\right)+1\right]
$$

and

$$
h_{2}\left(s_{1}\right)=C_{3}\left(L, \frac{s_{1}}{16}\right)\left[C_{3}\left(L, s_{1}\right) C_{17}\left(s_{1}\right)+\mathbf{E}^{0}\left[\tau_{B\left(0,2 s_{1}\right)}\right] \sup _{|y| \geq \frac{s_{1}}{4}} v(y)\right]+\sup _{|y| \geq \frac{s_{1}}{16}} v(y),
$$

where

$$
C_{17}\left(s_{1}\right):=K_{3}\left(s_{1}\right)+\frac{\mathbf{E}^{0}\left[\tau_{B\left(0,2 s_{1}\right)}\right]}{\left|B\left(0, \frac{s_{1}}{4}\right)\right|} K_{2}\left(\frac{s_{1}}{4}, \frac{s_{1}}{2}, s_{1}\right)^{2} .
$$

By (A1) there exists an absolute constant $c_{1}>0$ such that

$$
K_{2}\left(s_{1}, s_{2}, \infty\right) \leq c_{1} \text { and } K_{2}\left(\frac{s_{1}}{4}, \frac{s_{1}}{2}, s_{1}\right) \leq c_{1}
$$

and, from (2.4) and (A1) we get

$$
\sup _{|y| \geq \frac{s_{1}}{4}} v(y) \leq \sup _{|y| \geq \frac{s_{1}}{16}} v\left(y \leq c_{2} \frac{\Psi\left(1 / s_{1}\right)}{s_{1}^{d}}\right.
$$

This, together with (2.6), (2.9), (A3), and the doubling property of the function $\Psi$ yields

$$
h_{1}\left(s_{1}, s_{2}\right) \leq c_{3} \text { and } h_{2}\left(s_{1}\right) \leq c_{4} \frac{\Psi\left(1 / s_{1}\right)}{s_{1}^{d}},
$$

with constants $c_{3}, c_{4}>0$.

Let $s_{1} \geq 1$ arbitrary, and consider a non-negative function $f$ on $\mathbf{R}^{d}$ which is regular $(X, V)$-harmonic in a ball $B\left(x, s_{1}\right),|x| \geq r_{0}+s_{1}$. Then, by applying the argument in the 
second part of the proof of [29, Lem. 3.1] with the deterministic multiplicative functional $e^{-\eta t}$ replaced by $e^{-\int_{0}^{t} V\left(X_{s}\right) d s}$, we get

$$
\begin{aligned}
& f(y) \leq\left(\mathbf{E}^{y}\left[\int_{0}^{\tau}{ }_{B\left(x, \frac{s_{1}}{16}\right)} e^{-V_{*}(x) t} d t\right] \wedge \mathbf{E}^{y}\left[\tau_{B\left(x, \frac{s_{1}}{16}\right)}\right]\right) \\
& \times\left(h_{1}\left(s_{1}, s_{2}\right) \int_{|z-x|>s_{2}} f(z) v(z-x) d z+h_{2}\left(s_{1}\right) \int_{\frac{s_{1}}{8}<|x-z| \leq s_{2}} f(z) d z\right),
\end{aligned}
$$

for all $|y-x|<s_{1} / 32$ and $|x| \geq r_{0}+s_{1}$. Finally, by taking $s_{1}:=\eta|x|$ (then $\left.s_{2}=2 \eta|x|\right)$ with $|x| \geq r_{0} /(1-\eta)$ (so that $\left.|x| \geq r_{0}+\eta|x|\right)$, using (4.5) and the estimates

$$
\mathbf{E}^{y}\left[\int_{0}^{\tau_{B(x, \eta|x| / 16)}} e^{-V_{*}(x) t} d t\right] \leq \frac{c_{5}}{V_{*}(x)} \quad \text { and } \quad \mathbf{E}^{y}\left[\tau_{B(x, \eta|x| / 16)}\right] \leq \frac{c_{6}}{\Psi(1 /|x|)}
$$

(recall that $V_{*}(x) \geq 0$ and here we use the convention $1 / 0=+\infty$ ), we obtain the claimed bound

$$
f(y) \leq \frac{c_{7}}{V_{*}(x) \vee \Psi\left(\frac{1}{|x|}\right)}\left(\int_{|z-x|>2 \eta|x|} f(z) v(z-x) d z+\frac{\Psi\left(\frac{1}{|x|}\right)}{|x|^{d}} \int_{\frac{\eta|x|}{8}<|x-z| \leq 2 \eta|x|} f(z) d z\right),
$$

for $|x-y|<(\eta|x|) / 32$ and $|x| \geq r_{0} /(1-\eta)$. This completes the proof.

We single out two choices of $(X, V)$-harmonic functions of special interest below, for which the above estimate directly applies.

Corollary 4.1. Let $\left(X_{t}\right)_{t \geq 0}$ be a Lévy process with Lévy-Khintchin exponent $\psi$ as in (2.2) such that Assumptions (A1)-(A3) and (A4) are satisfied; specifically, let (A4) hold with some $r_{0}>0$. The following hold:

(1) If

$$
f(x)= \begin{cases}\mathbf{E}^{x}\left[e^{-\int_{0}^{\tau} \bar{B}\left(0, r_{0}\right)^{c}} V\left(X_{s}\right) d s\right] & \text { for } x \notin \bar{B}\left(0, r_{0}\right), \\ 1 & \text { for } x \in \bar{B}\left(0, r_{0}\right),\end{cases}
$$

then for every $|x| \geq 2 r_{0}$,

$$
f(x) \leq \frac{C_{4}}{V_{*}(x) \vee \Psi\left(\frac{1}{|x|}\right)}\left(\int_{|z-x|>\frac{|x|}{2}} f(z) v(z-x) d z+\frac{\Psi\left(\frac{1}{|x|}\right)}{|x|^{d}} \int_{\frac{|x|}{32}<|x-z| \leq \frac{|x|}{2}} f(z) d z\right) .
$$

(2) Let $\eta \in(0,1 / 4]$. If

$$
f(y)= \begin{cases}\mathbf{E}^{y\left[e^{-\int_{0}^{\tau} B(x, \eta|x|)^{c}} V\left(X_{s}\right) d s\right.} \varphi\left(X_{\tau_{B(x, \eta|x|)^{c}}}\right] & \text { for } y \in B(x, \eta|x|), \\ \varphi(y) & \text { for } y \notin B(x, \eta|x|),\end{cases}
$$


for some $x \notin B\left(0, r_{0} /(1-\eta)\right)$ and a function $\varphi: \mathbf{R}^{d} \rightarrow[0, \infty)$, not identically zero, then

$$
\begin{aligned}
f(y) \leq & \frac{C_{4}}{V_{*}(x) \vee \Psi\left(\frac{1}{|x|}\right)} \\
& \times\left(\int_{|z-x|>2 \eta|x|} f(z) v(z-x) d z+\frac{\Psi\left(\frac{1}{|x|}\right)}{|x|^{d}} \int_{\frac{\eta|x|}{8}<|x-z| \leq 2 \eta|x|} f(z) d z\right),
\end{aligned}
$$

whenever $|y-x|<\frac{\eta|x|}{32}$.

The above corollary is a straightforward consequence of Lemma 4.1. Indeed, by the strong Markov property of the underlying Lévy process, the function $f$ defined by (4.6) is regular $(X, V)$-harmonic in every ball $B(x,|x| / 4),|x| \geq 2 r_{0}$. Similarly, for given $x \notin \bar{B}\left(0, r_{0} /(1-\eta)\right)$, the function (4.7) is regular $(X, V)$-harmonic in a ball $B(x, \eta|x|)$.

We can now make use of the above estimates and the technical results obtained in the previous section to derive upper bounds for the zero-energy solutions for potentials satisfying (A4).

Theorem 4.1. Let $\left(X_{t}\right)_{t \geq 0}$ be a Lévy process with Lévy-Khintchin exponent $\psi$ as in (2.2) such that Assumptions (A1)-(A3) and (A4) hold; specifically, let (A4) hold with some $r_{0}>0$. Moreover, let $\varphi$ be a solution of (2.14). Then the following hold.

(1) If

$$
\lim _{|x| \rightarrow \infty} \frac{\Psi\left(\frac{1}{|x|}\right)}{V_{*}(x)}=0 \text { and } \int_{|x|>2 r_{0}} \frac{v(x)}{V_{*}(x)} d x<\infty,
$$

then there exists $C>0$ and $R \geq 2 r_{0}$ such that

$$
|\varphi(x)| \leq C\|\varphi\|_{\infty} \frac{v(x)}{V_{*}(x)}, \quad|x|>R .
$$

In particular, $\varphi \in L^{1}\left(\mathbf{R}^{d}\right)$.

(2) If

$$
\lim _{|x| \rightarrow \infty} \frac{\Psi\left(\frac{1}{|x|}\right)}{V_{*}(x)}=0 \text { and } \int_{|x|>2 r_{0}} \frac{v(x)}{V_{*}(x)} d x=\infty
$$

and

$$
\sup _{|x| \geq 2 r_{0}}\left[\frac{v(x)}{V_{*}(x)} \exp \left(\eta_{*} \int_{2 r_{0} \leq|z| \leq|x|} \frac{v(z)}{V_{*}(z)} d z\right)\right]<\infty,
$$

with $\eta_{*}:=C_{4} C_{6}$, then there exist $C>0$ and $R \geq 2 r_{0}$ such that

$$
|\varphi(x)| \leq C\|\varphi\|_{\infty} \frac{v(x)}{V^{*}(x)} \exp \left(\eta_{*} \int_{r_{0} \leq|z| \leq|x|} \frac{v(z)}{V_{*}(z)} d z\right), \quad|x|>R .
$$


(3) If $\liminf _{|x| \rightarrow \infty} \frac{\Psi\left(\frac{1}{|x|}\right)}{V_{*}(x)}>0, \varphi \geq 0$, and $\varphi \in L^{p}\left(\mathbf{R}^{d}\right)$ for some $p \in(1, \infty)$, then there exists $C>0$ such that

$$
\varphi(x) \leq C\|\varphi\|_{p}\left(\frac{\left(\int_{|z|>|x|} v(z)^{\frac{p}{p-1}} d z\right)^{\frac{p-1}{p}}}{\Psi\left(\frac{1}{|x|}\right)}+\frac{1}{|x|^{d / p}}\right), \quad|x|>2 r_{0} .
$$

Proof. By applying the resolvent formula (2.17) with any $\theta>0$ and $D=\bar{B}\left(0, r_{0}\right)^{c}$, and then on letting $\theta \downarrow 0$, we obtain

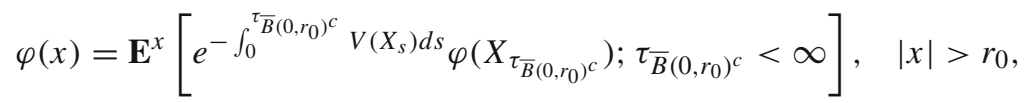

and hence

$$
|\varphi(x)| \leq\|\varphi\|_{\infty} \mathbf{E}^{x}\left[e^{-\int_{0}^{\tau \bar{B}\left(0, r_{0}\right)^{c}} V\left(X_{s}\right) d s}\right], \quad|x|>r_{0} .
$$

Next, let $f$ be the function defined in (4.6). With this we obtain

$$
|\varphi(x)| \leq\|\varphi\|_{\infty} f(x), \quad|x|>r_{0} .
$$

Choosing

$$
u(|x|):=C_{5} g(|x|), \quad w(|x|):=\Psi\left(\frac{1}{|x|}\right), \quad v(|x|):=V_{*}(|x|), \quad|x| \geq 2 r_{0},
$$

with $g$ as given in Assumption (A1), by Corollary 4.1 (1), we get for $|x|>2 r_{0}$

$$
f(x) \leq \frac{C_{4}}{v(|x|) \vee w(|x|)}\left(\int_{|z-x|>\frac{|x|}{2}} f(z) u(|z-x|) d z+\frac{w(|x|)}{|x|^{d}} \int_{\frac{|x|}{32}<|x-z| \leq \frac{|x|}{2}} f(z) d z\right) .
$$

To show parts (1)-(2) of the theorem note that, by assumption, $\lim _{|x| \rightarrow \infty} \Psi(1 /|x|) / v(|x|)$ $=0$, thus we may assume that $r_{0}$ is large enough such that $\Psi(1 /|x|) \vee v(|x|)=v(|x|)$, for $|x| \geq 2 r_{0}$. This means that the estimate (3.4) holds with constant $C_{13}=C_{4}$, radius $R_{0}:=2 r_{0} \vee 1$, and the above defined functions $u, w$ and $v$. Moreover, assumptions (3.1)(3.3) are also satisfied. Then the first two statements of the theorem follow directly by Lemma 3.1 and (4.9).

Next we prove the remaining part (3). First note that $\varphi \geq 0$ and similarly as in (4.8) we obtain

$$
\varphi(x)=\mathbf{E}^{x}\left[e^{-\int_{0}^{\tau} B(x,|x| / 4)} V\left(X_{s}\right) d s \varphi\left(X_{\tau_{B(x,|x| / 4)}}\right)\right], \quad|x|>2 r_{0} .
$$


Corollary 4.1 (2) gives the bound

$$
\varphi(x) \leq \frac{C_{4}}{\Psi\left(\frac{1}{|x|}\right)}\left(\int_{|z-x|>\frac{|x|}{2}} \varphi(z) v(z-x) d z+\frac{\Psi\left(\frac{1}{|x|}\right)}{|x|^{d}} \int_{\frac{|x|}{32}<|x-z| \leq \frac{|x|}{2}} \varphi(z) d z\right),
$$

for $|x|>2 r_{0}$. Finally, by Hölder inequality with suitable $p, q$,

$$
\begin{aligned}
\varphi(x) & \leq \frac{C_{4}}{\Psi\left(\frac{1}{|x|}\right)}\|\varphi\|_{p}\left(\int_{|z|>\frac{|x|}{2}} v(z)^{q} d z\right)^{1 / q}+\frac{c}{|x|^{d}}\|\varphi\|_{p}|B(0,|x| / 2)|^{1 / q} \\
& \leq c_{1}\|\varphi\|_{p}\left(\frac{\left(\int_{|z|>|x|} v(z)^{\frac{p}{p-1}} d z\right)^{\frac{p-1}{p}}}{\Psi\left(\frac{1}{|x|}\right)}+\frac{1}{|x|^{d / p}}\right), \quad|x|>2 r_{0},
\end{aligned}
$$

which completes the proof.

As it will be seen below, Theorem 4.1 (1)-(2) gives sharp upper bounds, provided $\varphi \geq 0$ (compare with the lower bounds in Theorem 4.3). We will now prove that if $\varphi$ is antisymmetric with respect to a given $(d-1)$-dimensional hyperplane $\pi$ in $\mathbf{R}^{d}$ with $\mathbf{0} \in \pi$, and has a definite sign on both of the corresponding half-spaces, then the decay rate in (1) of $|\varphi|$ at infinity far away from $\pi$ improves, while the upper bound in (3) remains unchanged. By rotating the coordinate system if necessary, we may assume that $\pi=\left\{x \in \mathbf{R}^{d}: x_{1}=0\right\}$. We make the assumption

$$
\begin{aligned}
& \varphi\left(\left(-x_{1}, x_{2}, \ldots, x_{d}\right)\right)=-\varphi\left(\left(x_{1}, x_{2}, \ldots, x_{d}\right)\right), x=\left(x_{1}, \ldots, x_{d}\right) \in \mathbf{R}^{d}, \\
& \quad \text { and } \varphi\left(\left(x_{1}, \ldots, x_{d}\right)\right) \geq 0 \text { whenever } x_{1}>0 .
\end{aligned}
$$

The next theorem deals with the case when $\varphi$ has no definite sign, but does satisfy (4.10).

Theorem 4.2. Let $\left(X_{t}\right)_{t \geq 0}$ be a Lévy process with Lévy-Khintchin exponent $\psi$ as in (2.2) such that Assumptions (A1)-(A3) and (A4) hold; specifically, let (A4) hold with some $r_{0}>0$. Suppose that there exist $C_{0}>0$ and $R_{0}>0$ such that

$$
\left|v\left(z_{1}\right)-v\left(z_{2}\right)\right| \leq C_{0} \frac{v\left(z_{2}\right)}{\left|z_{2}\right|}\left|z_{1}-z_{2}\right|, \quad\left|z_{1}\right| \geq\left|z_{2}\right| \geq R_{0},
$$

Moreover, let $\varphi$ be a solution of (2.14) such that (4.10) holds. The following hold:

(1) If $\lim _{|x| \rightarrow \infty} \frac{\Psi\left(\frac{1}{|x|}\right)}{V_{*}(x)}=0, \int_{|x|>2 r_{0}} \frac{v(x)}{V_{*}(x)} d x<\infty, \varphi \in L^{1}\left(\mathbf{R}^{d}\right)$ and there exists $a$ constant $C_{1}>0$ such that

$$
\frac{v(y)}{V_{*}(y)} \leq C_{1} \frac{v(x)}{V_{*}(x)}, \quad|y| \geq|x| / 2 \geq 2 r_{0},
$$

then there exists $C>0$ and $R \geq 2 r_{0} \vee 4 R_{0}$ such that for $\left|x_{1}\right|>R$ we have

$$
|\varphi(x)| \leq C\left(\|\varphi\|_{\infty} \vee\|\varphi\|_{1}\right) \frac{v(x)}{V_{*}(x)}\left(\frac{\Psi(1 /|x|)}{V_{*}(x)} \vee\left(\frac{1}{|x|} \int_{2 r_{0}<|z|<\frac{|x|}{2}}\left|z_{1}\right| \frac{v(z)}{V_{*}(z)} d z\right)\right) .
$$


(2) If $\liminf \inf _{|x| \rightarrow \infty} \frac{\Psi\left(\frac{1}{|x|}\right)}{V_{*}(x)}>0$ and $\varphi \in L^{p}\left(\mathbf{R}^{d}\right), p>1$, then there exists $C>0$ such that

$$
|\varphi(x)| \leq C\|\varphi\|_{p}\left(\frac{\left(\int_{|z|>|x|} v(z)^{\frac{p}{p-1}} d z\right)^{\frac{p-1}{p}}}{\Psi\left(\frac{1}{|x|}\right)}+\frac{1}{|x|^{d / p}}\right), \quad\left|x_{1}\right|>2 r_{0} .
$$

Proof. Using the resolvent formula (2.17) with $\theta>0$ and $D=\bar{B}(x,|x| / 4)^{c}$, and then letting $\theta \downarrow 0$, we obtain

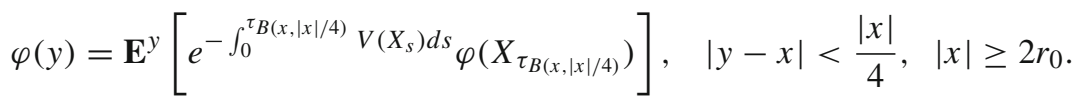

Denote $H_{+}:=\left\{z \in \mathbf{R}^{d}: z_{1}>0\right\}$ and $H_{-}:=\left\{z \in \mathbf{R}^{d}: z_{1}<0\right\}$. With this notation, by (4.10) we obtain

$$
\begin{aligned}
& \varphi(y)+\underbrace{\mathbf{E}^{y}\left[e^{-\int_{0}^{\tau_{B(x,|x| / 4)}} V\left(X_{s}\right) d s} \varphi_{-}\left(X_{\tau_{B(x,|x| / 4)}}\right) ; X_{\tau_{B(x,|x| / 4)}} \in H_{-} \cap B(0,|x| / 2)^{c}\right]}_{=: I_{1}(x, y)} \\
& =\underbrace{\mathbf{E}^{y}\left[e^{-\int_{0}^{\tau_{B(x,|x| / 4)}} V\left(X_{s}\right) d s} \varphi\left(X_{\tau_{B(x,|x| / 4)}}\right) ; X_{\tau_{B(x,|x| / 4)}} \in B(0,|x| / 2)\right]}_{=: I_{2}(x, y)} \\
& +\underbrace{\mathbf{E}^{y}\left[e^{-\int_{0}^{\tau_{B(x,|x| / 4)}} V\left(X_{s}\right) d s} \varphi_{+}\left(X_{\left.\tau_{B(x,|x| / 4)}\right)} ; X_{\tau_{B(x,|x| / 4)}} \in H_{+} \cap B(0,|x| / 2)^{c}\right]\right.}_{=: I_{3}(x, y)} .
\end{aligned}
$$

In particular,

$$
\varphi(x)=|\varphi(x)| \leq\left|I_{2}(x, x)\right|+I_{3}(x, x), \quad x_{1} \geq 2 r_{0} .
$$

To obtain (1), first we estimate $\left|I_{2}(x, x)\right|$. By the Ikeda-Watanabe formula (2.8), the change of variable $\left(z_{1}, \ldots, z_{d}\right)=z \mapsto \hat{z}=\left(-z_{1}, z_{2}, \ldots, z_{d}\right)$ in the inner integral over $H_{-} \cap B(0,|x| / 2)$, and (4.10) we obtain

$$
\begin{aligned}
I_{2}(x, x) & =\int_{B(x,|x| / 4)} G_{D}^{V}(x, d y) \int_{B(0,|x| / 2)} \varphi(z) v(y-z) d z \\
& =\int_{B(x,|x| / 4)} G_{D}^{V}(x, d y) \int_{H_{+} \cap B(0,|x| / 2)} \varphi(z)(v(y-z)-v(y-\hat{z})) d z .
\end{aligned}
$$


Thus by (4.10), (A1), and Theorem 4.1 (1) there exist $c_{1}, c_{2}, c_{3}>0$ and $\widetilde{R} \geq 2 r_{0}$ such that for $|x|>2 \widetilde{R} \vee 4 R_{0}$ it follows that

$$
\begin{aligned}
\left|I_{2}(x, x)\right| & \leq c_{1} \frac{v(x)}{|x|} G_{D}^{V}(x, B(x,|x| / 4)) \int_{B(0,|x| / 2)}|\varphi(z)|\left|z_{1}\right| d z \\
& \leq c_{2} \frac{v(x)}{|x| V_{*}(x)}\left(\int_{|z| \leq \widetilde{R}}|\varphi(z)|\left|z_{1}\right| d z+\int_{2 r_{0}<|z|<\frac{|x|}{2}} \frac{v(z)}{V_{*}(z)}\left|z_{1}\right| d z\right) \\
& \leq c_{3} \frac{v(x)}{V_{*}(x)} \frac{1}{|x|} \int_{2 r_{0}<|z|<\frac{|x|}{2}} \frac{v(z)}{V_{*}(z)}\left|z_{1}\right| d z .
\end{aligned}
$$

To estimate $I_{3}$, denote

$$
f_{x}(y)= \begin{cases}I_{3}(x, y) & \text { for } y \in B(x,|x| / 4), \\ \varphi(y) \mathbf{1}_{H_{+} \cap B(0,|x| / 2)^{c}}(y) & \text { for } y \notin B(x,|x| / 4),\end{cases}
$$

for every $|x| \geq 2 r_{0}$. The function $f_{x}(y)$ is regular $(X, V)$-harmonic in a ball $B(x,|x| / 2)$, and by Corollary 4.1 (2) it follows that

$$
f_{x}(y) \leq \frac{C_{4}}{V_{*}(x) \vee \Psi\left(\frac{1}{|x|}\right)}\left(\int_{|z-x|>\frac{|x|}{2}} f_{x}(z) v(z-x) d z+\frac{\Psi\left(\frac{1}{|x|}\right)}{|x|^{d}} \int_{\frac{|x|}{32}<|x-z| \leq \frac{|x|}{2}} f_{x}(z) d z\right)
$$

as long as $|y-x|<|x| / 128$ and $|x| \geq 2 r_{0}$. By the definition in (4.14) and the assumption that $\lim _{|x| \rightarrow \infty} \frac{\Psi\left(\frac{1}{|x|}\right)}{V_{*}(x)}=0$, there exists $R \geq 2 r_{0}$ such that the above estimate gives for $|x| \geq R$

$$
\begin{aligned}
I_{3}(x, x) \leq & \frac{C_{4}}{V_{*}(x)} \int_{\substack{|z-x|>\frac{|x|}{2} \\
|z|>\frac{|x|}{2}, z_{1}>0}} \varphi(z) v(z-x) d z+C_{4} \frac{\Psi\left(\frac{1}{|x|}\right)}{|x|^{d} V_{*}(x)} \int_{\frac{|x|}{4} \leq|x-z| \leq \frac{|x|}{2}} \varphi(z) d z \\
& +C_{4} \frac{\Psi\left(\frac{1}{|x|}\right)}{|x|^{d} V_{*}(x)} \int_{\frac{|x|}{32}<|x-z|<\frac{|x|}{4}} I_{3}(x, z) d z=: J_{1}(x)+J_{2}(x)+J_{3}(x) .
\end{aligned}
$$

The terms $J_{1}$ and $J_{2}$ can be estimated directly by using Theorem 4.1 (1), (4.12) and (2.4). Indeed, by increasing $R>0$ if necessary, we obtain

$$
J_{1}(x) \leq \frac{c_{4}}{V_{*}(x)}\left(\sup _{|y|<\frac{|x|}{2}} \frac{v(y)}{V_{*}(y)}\right) \int_{|z|>\frac{|x|}{2}} v(z) d z \leq c_{5} \frac{v(x)}{V_{*}(x)} \frac{\Psi\left(\frac{1}{|x|}\right)}{V_{*}(x)}, \quad|x| \geq R,
$$

and

$$
J_{2}(x) \leq c_{6} \frac{\Psi\left(\frac{1}{|x|}\right)}{V_{*}(x)}\left(\sup _{|y|<\frac{|x|}{2}} \frac{v(y)}{V_{*}(y)}\right) \leq c_{7} \frac{v(x)}{V_{*}(x)} \frac{\Psi\left(\frac{1}{|x|}\right)}{V_{*}(x)}, \quad|x| \geq R,
$$


for some positive constants $c_{4}, \ldots, c_{7}$. Moreover, there exists $c_{8}>0$ such that

$$
J_{3}(x) \leq c_{8} \frac{\Psi\left(\frac{1}{|x|}\right)}{V_{*}(x)} \sup _{y:|y-x|<\frac{|x|}{4}} I_{3}(x, y), \quad|x| \geq R .
$$

By (4.13) and Theorem 4.1 (1) we get for $|y-x|<\frac{|x|}{4}$ and $x_{1}>R$ that

$$
\begin{aligned}
& I_{3}(x, y) \\
& \quad \leq \varphi(y)+\mathbf{E}^{y}\left[e^{-\int_{0}^{\tau_{B(x,|x| / 4)}} V\left(X_{s}\right) d s} \varphi_{-}\left(X_{\tau_{B(x,|x| / 4)}}\right)\right] \\
& \quad \leq c_{9}\|\varphi\|_{\infty} \frac{v(y)}{V_{*}(y)}+\mathbf{E}^{y}\left[e^{-\int_{0}^{\tau_{B(x,|x| / 4)}} V\left(X_{s}\right) d s} \varphi_{-}\left(X_{\tau_{B(x,|x| / 4)}}\right)\right],
\end{aligned}
$$

and by one more use of (2.8),

$$
\begin{aligned}
& \mathbf{E}^{y}\left[e^{-\int_{0}^{\tau_{B(x,|x| / 4)}} V\left(X_{s}\right) d s} \varphi_{-}\left(X_{\tau_{B(x,|x| / 4)}}\right)\right] \\
& =c_{10} \int_{B(x,|x| / 4)} G_{B(x,|x| / 4)}^{V}(y, z) \int_{\left\{w: w_{1}<0\right\}} \varphi_{-}(w) v(w-z) d w d z \\
& \leq c_{11}\|\varphi\|_{1} \frac{v(x)}{V_{*}(x)}
\end{aligned}
$$

for $|y-x|<\frac{|x|}{4}$ and $x_{1}>R$. Due to (4.12), this means that

$$
\sup _{y:|y-x|<\frac{|x|}{4}} I_{3}(x, y) \leq c_{12}\left(\|\varphi\|_{\infty} \vee\|\varphi\|_{1}\right) \frac{v(x)}{V_{*}(x)}, \quad x_{1}>R .
$$

Putting together all the above estimates, we see that the upper bound in assertion (1) holds.

To establish (2), observe that similarly as above, for $|y-x|<\frac{|x|}{4}$ and $|x| \geq 2 r_{0}$ it follows that

$$
\varphi(y)=\mathbf{E}^{y}\left[e^{-\int_{0}^{\tau} B(x,|x| / 4)} V\left(X_{s}\right) d s\left(\varphi_{+}\left(X_{\tau_{B(x,|x| / 4)}}\right)-\varphi_{-}\left(X_{\left.\tau_{B(x,|x| / 4}\right)}\right)\right],\right.
$$

which yields for $|x| \geq 2 r_{0}$

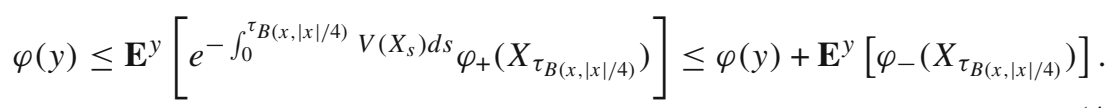

Define

$$
f_{x}(y)= \begin{cases}\mathbf{E}^{y}\left[e^{-\int_{0}^{\tau_{B(x,|x| / 4)}} V\left(X_{s}\right) d s} \varphi_{+}\left(X_{\left.\tau_{B(x,|x| / 4)}\right)}\right)\right. & \text { for } y \in B(x,|x| / 4), \\ \varphi_{+}(y) & \text { for } y \notin B(x,|x| / 4),\end{cases}
$$


for every $|x| \geq 2 r_{0}$. By the first inequality in (4.15) and Corollary 4.1 (2), we obtain

$$
\varphi(x) \leq \frac{C_{4}}{\Psi\left(\frac{1}{|x|}\right)}\left(\int_{|z-x|>\frac{|x|}{2}} \varphi_{+}(z) v(z-x) d z+\frac{\Psi\left(\frac{1}{|x|}\right)}{|x|^{d}} \int_{\frac{|x|}{32}<|x-z| \leq \frac{|x|}{2}} f_{x}(z) d z\right),
$$

for $x_{1}>2 r_{0}$, giving

$$
\varphi(x) \leq c_{13}\|\varphi\|_{p} \frac{\left(\int_{|z|>|x|} v(z)^{q} d z\right)^{1 / q}}{\Psi\left(\frac{1}{|x|}\right)}+\frac{c_{14}}{|x|^{d}} \int_{\frac{|x|}{32}<|x-z| \leq \frac{|x|}{2}} f_{x}(z) d z, \quad x_{1}>2 r_{0} .
$$

It suffices to estimate the latter integral. By the definition of the function $f_{x}$ and the second inequality in (4.15), we obtain

$$
\int f_{x}(z) d z \leq \int_{\frac{|x|}{32}<|x-z| \leq \frac{|x|}{2}} \int_{\frac{|x|}{32}<|x-z| \leq \frac{|x|}{2}}|\varphi(z)| d z+\int_{\frac{|x|}{32}<|x-z| \leq \frac{|x|}{4}} \mathbf{E}^{z}\left[\varphi_{-}\left(X_{\tau_{B(x,|x| / 4)}}\right)\right] d z .
$$

Similarly as above, (2.8) implies that for all $|z-x|<|x| / 4, x_{1}>2 r_{0}$, the estimate

$$
\begin{aligned}
\mathbf{E}^{z}\left[\varphi _ { - } \left(X_{\left.\left.\tau_{B(x,|x| / 4)}\right)\right]}\right.\right. & =c_{15} \int_{B(x,|x| / 4)} G_{B(x,|x| / 4)}(z, y) \int_{\left\{w: w_{1}<0\right\}} \varphi_{-}(w) v(w-y) d w d y \\
& \leq \frac{c_{16}}{\Psi\left(\frac{1}{|x|}\right)}\|\varphi\|_{p}\left(\int_{|y|>|x|} v(y)^{q} d y\right)^{1 / q},
\end{aligned}
$$

holds, and thus

$$
\int_{\frac{|x|}{32}<|x-z| \leq \frac{|x|}{2}} f_{x}(z) d z \leq c_{17}\|\varphi\|_{p}|x|^{d / q}+c_{18}\|\varphi\|_{p}|x|^{d} \frac{\left(\int_{|y|>|x|} v(y)^{q} d y\right)^{1 / q}}{\Psi\left(\frac{1}{|x|}\right)} .
$$

Inserting this estimate into (4.16), the claimed bound in (3) follows.

As it will be seen in specific cases in Sect. 6 below, by iterating the bounds in (1)-(2), we can often get the bound with $1 /|x|$ instead of $\frac{\Psi(1 /|x|)}{V_{*}(x)} \vee \frac{1}{|x|}$.

4.2. Lower bound. For a given potential $V$ satisfying Assumption (A4) denote

$$
V^{*}(x):=\sup _{|y| \geq \frac{|x|}{2}} V(y), \quad|x| \geq 2 r_{0}
$$

and

$$
\Lambda_{B(x,|x| / 2)}(x):=\mathbf{E}^{x}\left[\int_{0}^{\tau_{B(x,|x| / 2)}} e^{-V^{*}(x) t} d t\right], \quad|x| \geq 2 r_{0} .
$$


Clearly, $V^{*}(x)$ is a radial and non-increasing function. The auxiliary function $\Lambda_{V}$ has the suggestive meaning of lower envelope of the mean lifetime of the Lévy process under the potential $V$ in a ball $B(x,|x| / 2)$, that is,

$$
\Lambda_{B(x,|x| / 2)}(x) \leq \mathbf{E}^{x}\left[\int_{0}^{\tau_{B(x,|x| / 2)}} e^{-\int_{0}^{t} V\left(X_{s}\right) d s} d t\right], \quad|x| \geq 2 r_{0} .
$$

The first lemma gives a lower estimate on $\Lambda_{B(x,|x| / 2)}(x)$.

Lemma 4.2. Let $\left(X_{t}\right)_{t \geq 0}$ be a Lévy process with Lévy-Khintchin exponent $\psi$ as in (2.2) such that Assumptions (A1)-(A3) hold, and let $V$ be a potential satisfying (A4); specifically let (A4) hold with some $r_{0}>0$. Then there exists $C>0$ such that

$$
\Lambda_{B(x,|x| / 2)}(x) \geq \frac{C}{V_{*}(x) \vee \Psi\left(\frac{1}{|x|}\right)}, \quad|x| \geq 2 r_{0} .
$$

Proof. First notice that for every $\eta>0$,

$$
\Lambda_{B(x,|x| / 2)}(x) \geq \mathbf{E}^{0}\left[\int_{0}^{\eta} e^{-V^{*}(x) t} d t ; \tau_{B(0,|x| / 2)}>\eta\right], \quad|x| \geq 2 r_{0} .
$$

Thus

$$
\Lambda_{B(x,|x| / 2)}(x) \geq \frac{1-e^{-V^{*}(x) \eta}}{V^{*}(x)}\left(1-\mathbf{P}^{0}\left(\tau_{B(0,|x| / 2)} \leq \eta\right)\right), \quad|x| \geq 2 r_{0} .
$$

Moreover, by [45, eq. (3.2)] combined with (2.4), there exists $c_{1}>0$ such that for every $r, \eta>0$

$$
\mathbf{P}^{0}\left(\tau_{B(0, r)} \leq \eta\right) \leq c_{1} \eta \Psi\left(\frac{1}{r}\right)
$$

which gives

$$
1-\mathbf{P}^{0}\left(\tau_{B(0,|x| / 2)} \leq \eta\right) \geq 1-c_{1} \eta \Psi\left(\frac{1}{|x|}\right), \quad|x| \geq 2 r_{0} .
$$

Since the constant $c_{1}$ is uniform in $\eta>0$, we may take $\eta:=\frac{1}{2 c_{1} \Psi\left(\frac{1}{|x|}\right)}$, which implies that

$$
\Lambda_{B(x,|x| / 2)}(x) \geq \frac{1-\exp \left(-\frac{V^{*}(x)}{2 c_{1} \Psi\left(\frac{1}{|x|}\right)}\right)}{2 V^{*}(x)}, \quad|x| \geq 2 r_{0} .
$$

To conclude, it suffices to observe that if $V^{*}(x) \geq \Psi\left(\frac{1}{|x|}\right)$, then

$$
\Lambda_{B(x,|x| / 2)}(x) \geq \frac{1-e^{-\frac{1}{2 c_{1}}}}{2} \frac{1}{V^{*}(x)}, \quad|x| \geq 2 r_{0},
$$


and when $V^{*}(x) \leq \Psi\left(\frac{1}{|x|}\right)$

$$
\Lambda_{B(x,|x| / 2)}(x) \geq \frac{1}{2 c_{2}} \frac{1-\exp \left(-\frac{V^{*}(x)}{2 c_{1} \Psi\left(\frac{1}{|x|}\right)}\right)}{\frac{V^{*}(x)}{2 c_{1} \Psi\left(\frac{1}{|x|}\right)}} \frac{1}{\Psi\left(\frac{1}{|x|}\right)} \geq \frac{e^{\frac{1}{2 c_{1}}}}{2 c_{2}} \frac{1}{\Psi\left(\frac{1}{|x|}\right)}, \quad|x| \geq 2 r_{0},
$$

as required.

With this lemma we also have the following estimate.

Lemma 4.3. Let $\left(X_{t}\right)_{t \geq 0}$ be a Lévy process with Lévy-Khintchin exponent $\psi$ as in (2.2) such that Assumptions (A1)-(A3) hold and let $V$ be a potential satisfying (A4); specifically let (A4) hold with some $r_{0}>0$. Then for every positive solution $\varphi$ of (2.14) there exists $C>0$ such that

$$
\varphi(x) \geq \frac{C}{V_{*}(x) \vee \Psi\left(\frac{1}{|x|}\right)} \int_{\substack{|z|<|x| \\|z+x|<|z-x|}} \varphi(z) v(x-z) d z d y, \quad|x|>2 r_{0} .
$$

In particular,

$$
\varphi(x) \geq \frac{C}{C_{5}^{2} C_{6}}\left(\int_{B\left(0, r_{0}\right)} \varphi(z) d z\right) \frac{v(x)}{V_{*}(x) \vee \Psi\left(\frac{1}{|x|}\right)}, \quad|x|>2 r_{0} .
$$

Proof. By applying the resolvent formula (2.17) with any $\theta>0$ and $D=B(x,|x| / 2)$, and letting $\theta \downarrow 0$, we get

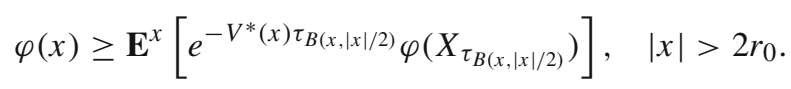

Then by the Ikeda-Watanabe formula (2.8) and (A1),

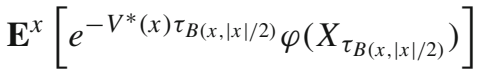

$$
\begin{aligned}
& \geq \int_{B(x,|x| / 2)} \int_{0}^{\infty} e^{-V^{*}(x) t} p_{B(x,|x| / 2)}(t, x, y) d t \int_{\substack{|z|<|x| \\
|z+x|<|z-x|}} \varphi(z) v(y-z) d z d y \\
& \geq c_{2} \Lambda_{B(x,|x| / 2)}(x) \int_{\substack{|z|<|x| \\
|z+x|<|z-x|}} \varphi(z) v(x-z) d z, \quad|x|>2 r_{0} .
\end{aligned}
$$

An application of Lemma 4.2 completes the proof of the first inequality. The second estimate is a direct consequence of the first.

We are now in the position to state the main theorem of this subsection.

Theorem 4.3. Let $\left(X_{t}\right)_{t \geq 0}$ be a Lévy process with Lévy-Khintchin exponent $\psi$ as in (2.2) such that Assumptions (A1)-(A3) hold and let $V$ be a potential satisfying (A4); specifically let (A4) hold with some $r_{0}>0$. Let $\varphi$ be a positive solution of (2.14). Then the following hold. 
(1) If $\lim _{|x| \rightarrow \infty} \frac{\Psi\left(\frac{1}{|x|}\right)}{V^{*}(x)}=0$ and $\int_{|x|>2 r_{0}} \frac{v(x)}{V^{*}(x)} d x<\infty$, then there exist $C>0$ and $R \geq 2 r_{0}$ such that

$$
\varphi(x) \geq C \frac{v(x)}{V^{*}(x)}, \quad|x|>R .
$$

(2) If $\lim _{|x| \rightarrow \infty} \frac{\Psi\left(\frac{1}{|x|}\right)}{V^{*}(x)}=0$ and $\int_{|x|>2 r_{0}} \frac{v(x)}{V^{*}(x)} d x=\infty$, then there exist $\eta^{*}, C>0$ and $R \geq 2 r_{0}$ such that

$$
\varphi(x) \geq C \frac{v(x)}{V^{*}(x)} \exp \left(\eta^{*} \int_{2 r_{0} \leq|y| \leq|x|} \frac{v(y)}{V^{*}(y)} d y\right), \quad|x|>R .
$$

(3) If $\lim \inf _{|x| \rightarrow \infty} \frac{\Psi\left(\frac{1}{|x|}\right)}{V^{*}(x)}>0$ and $\int_{|x|>2 r_{0}} \frac{v(x)}{\Psi\left(\frac{1}{|x|}\right)} d x<\infty$, then there exist $C>0$ and $R \geq 2 r_{0}$ such that

$$
\varphi(x) \geq C \frac{\nu(x)}{\Psi\left(\frac{1}{|x|}\right)}, \quad|x|>R .
$$

(4) If $\liminf \inf _{|x| \rightarrow \infty} \frac{\Psi\left(\frac{1}{|x|}\right)}{V^{*}(x)}>0$ and $\int_{|x|>2 r_{0}} \frac{v(x)}{\Psi\left(\frac{1}{|x|}\right)} d x=\infty$, then there exist $\eta^{*}, C>0$ and $R \geq 2 r_{0}$ such that

$$
\varphi(x) \geq C \frac{v(x)}{\Psi\left(\frac{1}{|x|}\right)} \exp \left(\eta^{*} \int_{2 r_{0} \leq|y| \leq|x|} \frac{v(y)}{\Psi\left(\frac{1}{|y|}\right)} d y\right), \quad|x|>R .
$$

Proof. As noted before, $\varphi \in C_{\mathrm{b}}\left(\mathbf{R}^{d}\right)$. First we prove (1)-(2). Let $u(|x|):=\frac{1}{C_{5}} g(|x|)$ and $v(|x|):=\mathbf{1}_{\left\{1 \leq|x| \leq 2 r_{0}\right\}} V^{*}\left(2 r_{0}\right)+\mathbf{1}_{\left\{1 \leq|x| \leq 2 r_{0}\right\}} V^{*}(|x|), \quad|x| \geq 1$, with $g$ given by Assumption (A1). By our assumptions and Lemma 4.3, there exist $c_{1}>0$ and $R \geq 2 r_{0}$ such that

$$
\varphi(x) \geq c_{1} \frac{1}{v(x)} \int_{\substack{|z|<|x| \\|z+x|<|z-x|}} \varphi(z) u(|x-z|) d z, \quad|x|>R .
$$

Since $0<\varphi \in C_{\mathrm{b}}\left(\mathbf{R}^{d}\right)$ and $\int_{|z+x|<|z-x|}|z|<|x|$ the same inequality holds also for this range of $|x|$. Thus the assumptions of Lemma 3.2 are satisfied with the functions $u$ and $v$. Hence there exist constants $c_{3}>0$ and $\eta^{*}>0$ for which the estimate

$$
\varphi(x) \geq c_{3} \frac{v(x)}{V^{*}(x)} \exp \left(\eta^{*} \int_{2 r_{0} \leq|y| \leq|x|} \frac{v(y)}{V^{*}(y)} d y\right), \quad|x|>R,
$$

holds. This implies (1) and (2). The proof of (3) and (4) follows by the same argument as above with the same $u(|x|)$ and $v(|x|):=\frac{1}{C_{1}} \Psi\left(\frac{1}{|x|}\right),|x| \geq 1$. 


\section{Decay of Zero-Energy Eigenfunctions for Potentials Negative at Infinity}

Now we turn to discussing the spatial decay properties of eigenfunctions of non-local Schrödinger operators with decaying potentials that are negative at infinity.

(A5) Let $V \in L^{\infty}\left(\mathbf{R}^{d}\right)$ be such that there exists $r_{0}>0$ and $C>0$ such that

$$
0 \leq-V(x) \leq C \Psi(1 /|x|), \quad|x| \geq r_{0} .
$$

Notice that under (A5) it follows that $V(x) \rightarrow 0$ as $|x| \rightarrow \infty$, i.e., $V$ is indeed a decaying potential. It also covers potentials with compact support such as potential wells.

We will now prove a counterpart of Theorem 4.2 (3) in the case when the potential is negative at infinity and the negative nodal domain of $\varphi$ is a subset of a given half-space. By rotating the coordinate system if necessary, without loss of generality we can assume that

$$
\text { there exists } l \in \mathbf{R} \text { such that } \operatorname{supp} \varphi_{-} \subset\left\{y \in \mathbf{R}^{d}: y_{1}<l\right\} .
$$

Theorem 5.1. Let $\left(X_{t}\right)_{t \geq 0}$ be a Lévy process with Lévy-Khintchin exponent $\psi$ as in (2.2) such that Assumptions (A1)-(A3) and (A5) hold; specifically, let (A5) hold with some $r_{0}>0$. Moreover, suppose that for every $\varepsilon \in(0,1)$ there exists $M \geq 1$ such that

$$
\Psi(r) \leq \varepsilon \Psi(M r), \quad r \in(0,1] .
$$

If $\varphi$ is a solution of (2.14) such that $\varphi \in L^{p}\left(\mathbf{R}^{d}\right)$, for some $p>1$, and (5.1) holds, then for every $\varepsilon \in(0,1)$ there exist $C>0$ and $R>3 r_{0}$ such that

$$
\varphi(x) \leq C\left(\|\varphi\|_{p} \vee\|\varphi\|_{\infty}\right)\left(\frac{\left(\int_{|y|>|x|} v(y)^{\frac{p}{p-(1+\varepsilon)}} d y\right)^{\frac{p-(1+\varepsilon)}{p}}}{\Psi(1 /|x|)}+\frac{1}{|x|^{\frac{d}{p}}}\right)^{1-\varepsilon}, \quad x_{1} \geq R .
$$

Moreover, if $\varphi\left(l+x_{1}, x_{2}, \ldots, x_{d}\right)=-\varphi\left(l-x_{1}, x_{2}, \ldots, x_{d}\right), x \in \mathbf{R}^{d}$, with $l$ given by (5.1), then there exists $\widetilde{R}>3 r_{0} \vee|l|$ such that the same upper bound is true for $\varphi(x)$ replaced by $|\varphi(x)|$, whenever $\left|x_{1}\right| \geq \widetilde{R}$.

Proof. For $\eta \in(0,1 / 4]$ we denote $D_{\eta}:=B(x, \eta|x|), x \in \mathbf{R}^{d}$. First note that there exists $\eta \in(0,1 / 4]$ such that

$$
\sup _{|x|>\frac{r_{0} \vee 1}{1-\eta}} \mathbf{E}^{x}\left[e^{\int_{0}^{\tau} D_{\eta}}\left|V\left(X_{t}\right)\right| d t\right]<\infty .
$$

Indeed, by (2.9) and Assumption (A5), there is $c>0$ such that

$\mathbf{E}^{x}\left[\int_{0}^{\tau_{D_{\eta}}}\left|V\left(X_{t}\right)\right| d t\right] \leq \sup _{y \in D_{\eta}}|V(y)| \mathbf{E}^{x}\left[\tau_{D_{\eta}}\right] \leq c \frac{\Psi(1 /((1-\eta)|x|))}{\Psi(1 /(\eta|x|))}, \quad|x|>\frac{r_{0}}{1-\eta}$,

and, by using (5.2), we can derive from the above that

$$
\sup _{|x|>\frac{r_{0} \vee 1}{1-\eta}} \mathbf{E}^{x}\left[\int_{0}^{\tau_{D_{\eta}}}\left|V\left(X_{t}\right)\right| d t\right]<1
$$


for sufficiently small $\eta$. Similarly, by the fact that $V \in L^{\infty}\left(\mathbf{R}^{d}\right)$, cf. (A5), and using (2.9),

$$
\sup _{|x| \leq \frac{r_{0} \vee 1}{1-\eta}} \mathbf{E}^{x}\left[\int_{0}^{\tau_{D \eta}}\left|V\left(X_{t}\right)\right| d t\right]<1
$$

for $\eta$ small enough. Hence, by Khasminskii's Lemma we see that there exists $\eta \in\left(0, \frac{1}{4}\right]$ such that (5.3) holds. Next, by applying (2.17) for $\theta>0$ we obtain

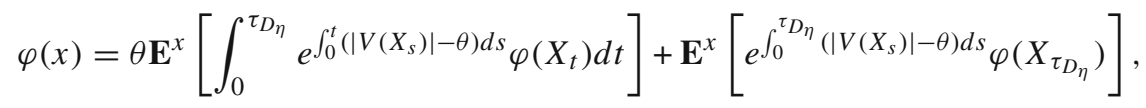

for $|x|>\frac{r_{0} \vee 1}{1-\eta}$. Letting $\theta \rightarrow \infty$ this gives

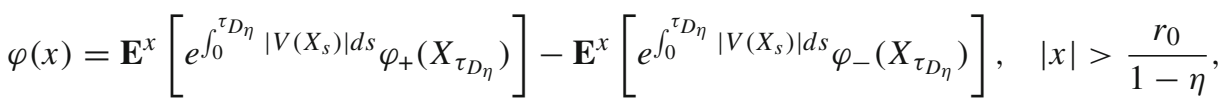

with $\eta$ specified above, where $\varphi_{ \pm}$denotes the positive and negative parts of $\varphi$, respectively. (We note that passing to the limit is possible due to (5.3) and dominated convergence.) In particular,

$$
\varphi(x) \leq \mathbf{E}^{x}\left[e^{\int_{0}^{\tau} D_{\eta}}\left|V\left(X_{s}\right)\right| d s \varphi_{+}\left(X_{\tau_{D_{\eta}}}\right)\right], \quad|x|>\frac{r_{0} \vee 1}{1-\eta},
$$

and

$$
\mathbf{E}^{x}\left[\varphi_{+}\left(X_{\tau_{D_{\eta}}}\right)\right] \leq \varphi(x)+\mathbf{E}^{x}\left[e^{\int_{0}^{\tau D_{\eta}}\left|V\left(X_{s}\right)\right| d s} \varphi_{-}\left(X_{\tau_{D_{\eta}}}\right)\right], \quad|x|>\frac{r_{0} \vee 1}{1-\eta} .
$$

Furthermore, by Hölder inequality with $\widetilde{p}, \widetilde{q}>1$ such that $1 / \widetilde{p}+1 / \widetilde{q}=1$, we obtain

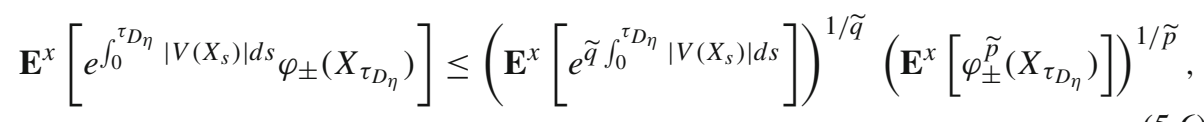

for $|x|>\frac{r_{0} \vee 1}{1-\eta}$. Again, by Khasminskii's Lemma and by decreasing $\eta$ (dependent on $\widetilde{q}$ ) if necessary, we obtain that

$$
C_{\eta, \tilde{q}}:=\sup _{x \in \mathbf{R}^{d}}\left(\mathbf{E}^{x}\left[e^{\tilde{q} \int_{0}^{\tau} D_{\eta}}\left|V\left(X_{s}\right)\right| d s\right]\right)^{1 / \widetilde{q}}<\infty .
$$

Therefore, by (5.4) and (5.6), it follows that

$$
\varphi(x) \leq C_{\eta, \widetilde{q}}\left(\mathbf{E}^{x}\left[\varphi_{+}^{\widetilde{p}}\left(X_{\tau_{D_{\eta}}}\right)\right]\right)^{1 / \widetilde{p}}, \quad|x|>\frac{r_{0} \vee 1}{1-\eta},
$$

and

$$
\mathbf{E}^{x}\left[e^{\int_{0}^{\tau} D_{\eta}}\left|V\left(X_{s}\right)\right| d s \varphi_{-}\left(X_{\tau_{D_{\eta}}}\right)\right] \leq C_{\eta, \widetilde{q}}\left(\mathbf{E}^{x}\left[\varphi_{-}^{\tilde{p}}\left(X_{\tau_{D_{\eta}}}\right)\right]\right)^{1 / \widetilde{p}}, \quad|x|>\frac{r_{0} \vee 1}{1-\eta} .
$$


Fix now an arbitrarily small $\varepsilon>0$ and choose $\eta=\eta(\varepsilon) \in\left(0, \frac{1}{4}\right]$ small enough such that (5.7) holds with $\widetilde{p}=1+\varepsilon$ and $C_{\eta, \widetilde{q}}<\infty$, where $\widetilde{q}=1+1 / \varepsilon$. In particular,

$$
\varphi(x)^{1+\varepsilon} \leq C_{\eta, 1+1 / \varepsilon} \mathbf{E}^{x}\left[\varphi_{+}^{1+\varepsilon}\left(X_{\tau_{D_{\eta}}}\right)\right], \quad|x|>\frac{r_{0} \vee 1}{1-\eta} .
$$

We define $f_{x}(y)=\varphi_{+}^{1+\varepsilon}(y)$ for $y \in D_{\eta}^{c}$, and $f_{x}(y)=\mathbf{E}^{y}\left[\varphi_{+}^{1+\varepsilon}\left(X_{\tau_{D_{\eta}}}\right)\right]$ for $y \in D_{\eta}$. Clearly, $f_{x}$ is $X$-harmonic in $D_{\eta}$. Recall that both $D_{\eta}=B(x, \eta|x|)$ and $f_{x}$ depend on the position $x$. By Corollary $4.1(2)$, there exists $c_{1}=c_{1}(\eta)$ such that

$$
\begin{aligned}
f_{x}(x) \leq & \frac{c_{1}}{\Psi(1 /|x|)} \int_{|y-x|>2 \eta|x|} f_{x}(y) v(x-y) d y \\
& +\frac{c_{1}}{|x|^{d}} \int_{\frac{\eta}{8}|x|<|y-x|<2 \eta|x|} f_{x}(y) d y, \quad|x| \geq 2\left(r_{0} \vee 1\right) .
\end{aligned}
$$

Denote the summands at the right hand side above by $I_{1}(x)$ and $I_{2}(x)$, respectively. Again, by applying Hölder inequality with $\widetilde{p}=p /(1+\varepsilon)$ and $\widetilde{q}=p /(p-(1+\varepsilon))$, and using Assumption (A1), it is seen that there exists $c_{2}=c_{2}(\varepsilon, p)>0$ such that

$$
\begin{aligned}
I_{1}(x) & \leq \frac{c_{1}}{\Psi(1 /|x|)}\left(\int_{\mathbf{R}^{d}} \varphi_{+}^{p}(y) d y\right)^{\frac{1+\varepsilon}{p}}\left(\int_{|y|>2 \eta|x|} v(y)^{\frac{p}{p-(1+\varepsilon)}} d y\right)^{\frac{p-(1+\varepsilon)}{p}} \\
& \leq c_{2}\|\varphi\|_{p}^{1+\varepsilon} \frac{\left(\int_{|y|>|x|} v(y)^{\frac{p}{p-(1+\varepsilon)}} d y\right)^{\frac{p-(1+\varepsilon)}{p}}}{\Psi(1 /|x|)}, \quad|x| \geq 2\left(r_{0} \vee 1\right) \vee \frac{1}{2 \eta}
\end{aligned}
$$

Moreover, by the definition of $f_{x},(5.5)$, and (5.8) applied with $\tilde{p}=p, \widetilde{q}=q$,

$$
\begin{aligned}
I_{2}(x) \leq & \frac{c_{1}}{|x|^{d}}\left(\int_{\eta|x|<|y-x|<2 \eta|x|}|\varphi(y)|^{1+\varepsilon} d y+\int_{\frac{\eta}{8}|x|<|y-x| \leq \eta|x|} \mathbf{E}^{y}\left[\varphi_{+}^{1+\varepsilon}\left(X_{\tau_{D_{\eta}}}\right)\right] d y\right) \\
\leq & \frac{c_{1}}{|x|^{d}}\left(\int_{\eta|x|<|y-x|<2 \eta|x|}|\varphi(y)|^{1+\varepsilon} d y+\|\varphi\|_{\infty}^{\varepsilon} \int_{\frac{\eta}{8}|x|<|y-x| \leq \eta|x|} \varphi(y) d y\right. \\
& \left.+C_{\eta, q}\|\varphi\|_{\infty}^{\varepsilon} \int_{\frac{\eta}{8}|x|<|y-x| \leq \eta|x|}\left(\mathbf{E}^{y}\left[\varphi_{-}^{p}\left(X_{\tau_{D_{\eta}}}\right)\right]\right)^{1 / p} d y\right), \quad|x| \geq 3\left(r_{0} \vee 1\right),
\end{aligned}
$$

and by a further application of the Hölder inequality with $\widetilde{p}=p /(1+\varepsilon)$ and $\widetilde{q}=$ $p /(p-(1+\varepsilon))$ to the first integral, and with $p, q$ to the second and third, we get

$$
I_{2}(x) \leq \frac{c_{3}\|\varphi\|_{p}^{1+\varepsilon}}{|x|^{\frac{(1+\varepsilon) d}{p}}}+\frac{c_{4}\|\varphi\|_{\infty}^{\varepsilon}}{|x|^{\frac{d}{p}}}\left(\|\varphi\|_{p}+\left(\int_{|y-x| \leq \eta|x|} \mathbf{E}^{y}\left[\varphi_{-}^{p}\left(X_{\tau_{D_{\eta}}}\right)\right] d y\right)^{1 / p}\right),
$$

for $|x| \geq 3\left(r_{0} \vee 1\right)$, with some $c_{3}=c_{3}(\varepsilon, p)$ and $c_{4}=c_{4}(\varepsilon, p)$. It suffices to estimate the latter integral. By (2.8), Assumption (A1), (5.1) and (2.9), for every $y \in B(x, \eta|x|)$ and $x \in \mathbf{R}^{d}$ such that $x_{1}>3\left(r_{0} \vee 1\right)$, it follows that 


$$
\begin{aligned}
\mathbf{E}^{y}\left[\varphi_{-}^{p}\left(X_{\tau_{D_{\eta}}}\right)\right] & =c_{5} \int_{D_{\eta}} G_{D_{\eta}}(y, z) \int_{D_{\eta}^{c}} \varphi_{-}^{p}(w) v(w-z) d w d z \\
& =c_{5} \int_{D_{\eta}} G_{D_{\eta}}(y, z) \int_{\left\{w: w_{1}<l\right\}} \varphi_{-}^{p}(w) v(w-z) d w d z \\
& \leq c_{6} \mathbf{E}^{y}\left[\tau_{D_{\eta}}\right]\|\varphi\|_{p}^{p} v(x) \\
& \leq c_{7}\|\varphi\|_{p}^{p} \frac{v(x)}{\Psi\left(\frac{1}{\eta|x|}\right)} \leq c_{8}\|\varphi\|_{p}^{p} \frac{v(x)}{\Psi\left(\frac{1}{|x|}\right)},
\end{aligned}
$$

with some $c_{5}, \ldots, c_{8}$, possibly depending on $\varepsilon$ via $\eta$. Thus

$$
I_{2}(x) \leq \frac{c_{3}\|\varphi\|_{p}^{1+\varepsilon}}{|x|^{\frac{(1+\varepsilon) d}{p}}}+\frac{c_{9}\|\varphi\|_{\infty}^{\varepsilon}\|\varphi\|_{p}}{|x|^{\frac{d}{p}}}\left(1+\left(\frac{v(x)|x|^{d}}{\Psi\left(\frac{1}{|x|}\right)}\right)^{1 / p}\right), \quad x_{1}>3\left(r_{0} \vee 1\right),
$$

with $c_{9}=c_{9}(\varepsilon, p)$. Note also that under (A1) there exists $c_{10}>0$ such that $v(x) \leq$ $c_{10} \Psi\left(\frac{1}{|x|}\right)|x|^{-d},|x| \geq 3\left(r_{0} \vee 1\right)$. By putting all the above estimates together, we see that there exists a constant $c_{11}=c_{11}(\varepsilon, p)$ such that

$$
\varphi(x) \leq c_{11}\left(\|\varphi\|_{p} \vee\|\varphi\|_{\infty}\right)\left(\frac{\left(\int_{|y|>|x|} v(y)^{\frac{p}{p-(1+\varepsilon)}} d y\right)^{\frac{p-(1+\varepsilon)}{p}}}{\Psi(1 /|x|)}+\frac{1}{|x|^{\frac{d}{p}}}\right)^{1 /(1+\varepsilon)},
$$

whenever $x_{1}>3\left(r_{0} \vee 1\right) \vee \frac{1}{2 \eta}$, which is the first claimed bound. The second statement of the theorem follows from this by the antisymmetry argument.

A further discussion of the potentials negative at infinity in some specific cases will be made at the end of Sect. 6.1 below.

\section{Specific Cases and Decay Mechanisms}

6.1. Isotropic and anisotropic fractional Schrödinger operators. Let $L^{(\alpha)}, \alpha \in(0,2)$, be a family of self-adjoint pseudo-differential operators determined by their Fourier transforms

$$
\begin{aligned}
& \widehat{L^{(\alpha)}} f(\xi)=-\psi^{(\alpha)}(\xi) \widehat{f}(\xi), \quad \xi \in \mathbf{R}^{d}, \\
& f \in \operatorname{Dom}\left(L^{(\alpha)}\right)=\left\{g \in L^{2}\left(\mathbf{R}^{d}\right): \psi^{(\alpha)} \widehat{g} \in L^{2}\left(\mathbf{R}^{d}\right)\right\},
\end{aligned}
$$

where

$$
\psi^{(\alpha)}(\xi)=\int_{\mathbf{R}^{d} \backslash\{0\}}(1-\cos (\xi \cdot z)) v^{(\alpha)}(z) d z .
$$

Here we take $v^{(\alpha)}(x)=g(x /|x|)|x|^{-d-\alpha}, d \geq 1$, where the function $g: \mathbb{S}_{d-1} \rightarrow$ $(0, \infty)$, with the $(d-1)$-dimensional unit sphere $\mathbb{S}_{d-1}$ centered in the origin, is such that $g(\theta)=g(-\theta)$ and $c_{1} \leq g(\theta) \leq c_{2}$, for every $\theta \in \mathbb{S}_{d-1}$, with finite positive 
constants $c_{1}, c_{2}$ (cf. (2.2)). Clearly, every $v^{(\alpha)}(z) d z$ is a symmetric Lévy measure on $\mathbf{R}^{d} \backslash\{0\}$ such that

$$
v^{(\alpha)}(x) \asymp|x|^{-d-\alpha}, \quad x \in \mathbf{R}^{d} \backslash\{0\} .
$$

In particular, $\int_{\mathbf{R}^{d} \backslash\{0\}} v^{(\alpha)}(z) d z=\infty$ and Assumption (A1) holds. Also, one can easily check that $\psi^{(\alpha)}(\xi) \asymp|\xi|^{\alpha}$. From this we can easily see that the maximal function $\Psi$ of the symbol $\psi$ defined in (2.3) satisfies

$$
\Psi(r) \asymp r^{\alpha}, \quad r>0 .
$$

When the spherical density $g$ is non-trivial, the operator $L^{(\alpha)}$ is often called an anisotropic fractional Laplacian of order $\alpha / 2$, and the corresponding stochastic process generated by it is an anisotropic $\alpha$-stable Lévy process. When $g \equiv C_{d, \alpha}$ for a constant $C_{d, \alpha}>0$, the operator $L^{(\alpha)}=-(-\Delta)^{\alpha / 2}$ is given by the usual isotropic fractional Laplacian, generating a rotationally symmetric Lévy process.

Note that, by symmetry, for every $t>0$ it follows that

$$
\sup _{x \in \mathbf{R}^{d}} p(t, x)=p(t, 0)=\int_{\mathbf{R}^{d}} e^{-t \psi(\xi)} d \xi<c_{3} t^{-d / \alpha}, \quad t>0,
$$

and, as proven in [19],

$$
p(t, x) \leq c_{4} t|x|^{-d-\alpha}, \quad t>0, \quad x \in \mathbf{R}^{d} \backslash\{0\} .
$$

Then (6.4) gives (A2), and (A3) follows by a combination of (6.4), (6.5) and [29, Lem. 2.2].

First we consider potentials that are positive at infinity in the sense of (A4) and look at positive solutions of (2.15).

Theorem 6.1. Let $L^{(\alpha)}, 0<\alpha<2$, be a pseudo-differential operator determined by (6.1) and $V$ be an $X$-Kato class potential for which there exists $r_{0}>0$ such that $V(x)>0$ and $V(x) \asymp|x|^{-\beta}$, for $|x| \geq r_{0}$, with some $\beta>0$. Suppose that there exists a positive function $\varphi \in C_{\mathrm{b}}\left(\mathbf{R}^{d}\right)$ which is a solution of (2.15). Then the following hold:

(1) If $\beta<\alpha$, then there exist constants $C_{1}, C_{2}>0$ such that

$$
\frac{C_{1}}{(1+|x|)^{d+\alpha-\beta}} \leq \varphi(x) \leq \frac{C_{2}}{(1+|x|)^{d+\alpha-\beta}}, \quad x \in \mathbf{R}^{d} .
$$

In particular, $\varphi \in L^{p}\left(\mathbf{R}^{d}\right)$, for every $p \geq 1$.

(2) If $\beta \geq \alpha$, then there exist $\gamma \in(0,1)$ and a constant $C_{3}>0$ such that

$$
\varphi(x) \geq \frac{C_{3}}{(1+|x|)^{d-\gamma}}, \quad x \in \mathbf{R}^{d} .
$$

In particular, $\varphi \notin L^{p}\left(\mathbf{R}^{d}\right)$, for every $p \in\left[1, \frac{d}{d-\gamma}\right]$. On the other hand, if $\varphi \in L^{p}\left(\mathbf{R}^{d}\right)$ for some $p>1$, then there exists $C_{4}>0$ such that

$$
\varphi(x) \leq \frac{C_{4}}{(1+|x|)^{d / p}}, \quad x \in \mathbf{R}^{d} .
$$

In particular, if $\varphi \in L^{p}\left(\mathbf{R}^{d}\right)$ with $p=\frac{d}{d-\gamma-\varepsilon}$ for some $\varepsilon \in(0,1)$, then there exists $C_{4}>0$ such that

$$
\varphi(x) \leq \frac{C_{4}}{(1+|x|)^{d-\gamma-\varepsilon}}, \quad x \in \mathbf{R}^{d} .
$$


Proof. Due to (6.2) and (6.3), the upper bounds in (1)-(2) follow directly from Theorem 4.1 (1) and (3). The corresponding lower estimates are a consequence of Theorem 4.3 (1) and (4), respectively.

This has the following implication.

Corollary 6.1. Under the assumptions of Theorem 6.1 it follows that the function $\varphi$ belongs to $L^{1}\left(\mathbf{R}^{d}\right)$ if and only if $\alpha>\beta$.

Remark 6.1. Let $V$ be a potential positive at infinity, and $V(x) \asymp|x|^{-\beta}$ as $|x| \rightarrow \infty$, and consider the fractional Laplacian $(-\Delta)^{\alpha / 2}, 0<\alpha<2$. Although the constants are hard to control in sufficient detail, a calculation using the above estimates shows that if $\beta \geq \alpha$ and $\frac{C_{1} C_{15}}{C_{5} C_{10}} \geq d$, then zero is not an eigenvalue of $(-\Delta)^{\alpha / 2}+V$. We note that $C_{10}$ and $C_{15}$ play the more important role here, giving some best constants involving the jump doubling domination rate and another ratio related to jump activity. Also, from Theorem 6.1(2) we see that whenever $(0,1) \ni \gamma \geq \frac{d}{2}$, which may occur when $d=1$, the operator $H$ has no zero eigenvalue.

It can already be seen from the above theorem that there is a transition in the localization properties of $\varphi$ when $\alpha>\beta$ changes to $\alpha \leq \beta$. For a closer understanding of this transition around $\alpha \approx \beta$, we consider a more refined class of potentials.

Theorem 6.2. Let $L^{(\alpha)}, 0<\alpha<2$, be a pseudo-differential operator determined by (6.1) and $V$ be an $X$-Kato class potential for which there exists $r_{0}>0$ such that $V(x)>0$ and $V(x) \asymp|x|^{-\alpha}(\log |x|)^{\delta}$, for $|x| \geq r_{0}$, with some $\delta>0$. Suppose that there exists $a$ positive function $\varphi \in C_{\mathrm{b}}\left(\mathbf{R}^{d}\right)$ which is a solution of (2.15). Then the following hold.

(1) If $\delta>1$, then there exist constants $C_{1}, C_{2}>0$ such that

$$
\frac{C_{1}}{(1+|x|)^{d}(\log (1+|x|))^{\delta}} \leq \varphi(x) \leq \frac{C_{2}}{(1+|x|)^{d}(\log (1+|x|))^{\delta}}, \quad x \in \mathbf{R}^{d} .
$$

In particular, $\varphi \in L^{p}\left(\mathbf{R}^{d}\right)$, for every $p \geq 1$.

(2) If $\delta=1$, then there exist $0<\gamma_{1} \leq 1 \leq \gamma_{2}$ and constants $C_{4}, C_{5}>0$ such that

$$
\frac{C_{1}}{(1+|x|)^{d}(\log (1+|x|))^{1-\gamma_{1}}} \leq \varphi(x) \leq \frac{C_{2}}{(1+|x|)^{d}(\log (1+|x|))^{1-\gamma_{2}}}, \quad x \in \mathbf{R}^{d} .
$$

In particular, $\varphi \in L^{p}\left(\mathbf{R}^{d}\right)$ for every $p>1$, but $\varphi \notin L^{1}\left(\mathbf{R}^{d}\right)$.

(3) If $\delta \in(0,1)$, then there exist $0<\gamma_{1} \leq 1 \leq \gamma_{2}$ and constants $C_{6}, C_{7}>0$ such that

$$
C_{6} \frac{e^{\frac{\gamma_{1}}{1-\delta}(\log |x|)^{1-\delta}}}{(1+|x|)^{d}(\log (1+|x|))^{\delta}} \leq \varphi(x) \leq C_{7} \frac{e^{\frac{\gamma_{2}}{1-\delta}(\log |x|)^{1-\delta}}}{(1+|x|)^{d}(\log (1+|x|))^{\delta}}, \quad x \in \mathbf{R}^{d} .
$$

In particular, $\varphi \in L^{p}\left(\mathbf{R}^{d}\right)$, for every $p>1$, but $\varphi \notin L^{1}\left(\mathbf{R}^{d}\right)$.

(4) If $\delta \leq 0$, then exactly the same bounds and $L^{p}$-properties hold as in (2) of Theorem 6.1 .

Proof. Similarly as above, the upper bounds in (1)-(4) follow by an application of the estimates in Theorem 4.1 (1)-(3); specifically, both upper estimates (2) and (3) result from assertion (2) of this theorem. The corresponding lower estimates are consequences of the respective bounds in (1), (2), and (4) of Theorem 4.3. 
From the results above it is seen that the possible localization properties of the positive zero-energy eigenfunctions or zero-resonances for decaying potentials positive at infinity splits naturally into disjoint regimes representing the following three different scenarios. (For the simplicity of the discussion here, we assume that $V$ is a potential that is positive at infinity and regular enough so that $V(x) \asymp V^{*}(x) \asymp V_{*}(x)$ far away from the origin). Let $r_{0}>0$ be large enough, and define

$$
h(r)=\int_{r_{0}<|x|<r} \frac{v(x)}{V(x)} d x \text { and } \tilde{h}(r)=\int_{r_{0}<|x|<r} \frac{v(x)}{\Psi(1 /|x|)} d x, \quad r>r_{0} .
$$

Clearly, $h$ and $\tilde{h}$ are bounded functions on $\left(r_{0}, \infty\right)$ if and only if the ratios $v / V$ and $\nu / \Psi(1 /|x|)$, respectively, are integrable at infinity. The following situations occur:

- Scenario (1): If $\lim _{|x| \rightarrow \infty} \frac{\Psi(1 /|x|)}{V(x)}=0$ and the ratio $\frac{v(x)}{V(x)}$ is integrable at infinity, then

$$
\varphi(x) \asymp \frac{v(x)}{V(x)},
$$

for large enough $|x|$. In particular, $\varphi \in L^{1}\left(\mathbf{R}^{d}\right)$. Clearly, in this case the corresponding function $h$ is bounded.

- Scenario (2): If $\lim _{|x| \rightarrow \infty} \frac{\Psi(1 /|x|)}{V(x)}=0$ and the integrability of $\frac{v(x)}{V(x)}$ at infinity breaks down, then $h(r) \rightarrow \infty$ as $r \rightarrow \infty$. Hence (1) is no longer true and the function $h$ contributes into the behaviour of $\varphi$ at infinity like

$$
C_{1} \frac{v(x)}{V(x)} e^{\gamma_{1} h(|x|)} \leq \varphi(x) \leq C_{2} \frac{\nu(x)}{V(x)} e^{\gamma_{2} h(|x|)},
$$

for large enough $x$, with some $0<\gamma_{1} \leq 1 \leq \gamma_{2}$ and $C_{1}, C_{2}>0$. Observe that Scenario (1) differs from (2) by the boundedness of $h$.

- Scenario (3): If $\liminf |x| \rightarrow \infty \frac{\Psi(1 /|x|)}{V(x)}>0$, then the fall-off rate of $\varphi$ at infinity rapidly decreases so that

$$
\varphi(x) \geq C_{1} \frac{\nu(x)}{\Psi(1 /|x|)} e^{\gamma \widetilde{h}(|x|)}
$$

for large $x$, with some $\gamma, C_{1}>0$. Clearly, in case $\tilde{h}$ is bounded, it does not contribute to the above lower rate.

Now we revisit the examples in (1.1) in the situation of Theorems 6.1 and 6.2.

Example 6.1. Let $\alpha \in(0,2), d \geq 1$, and $L^{(\alpha)}=-(-\Delta)^{\alpha / 2}$. In this case

$$
v(x)=\frac{C(d, \alpha)}{|x|^{d+\alpha}} \quad \text { and } \quad \psi(x)=\Psi(|x|)=|x|^{\alpha} .
$$

To have $\varphi_{\kappa}>0$, we consider $l=0$ in (1.1), and to have positive potentials at infinity, we consider $\kappa \in\left(\frac{d-\alpha}{2}, \frac{d+\alpha}{2}\right), \alpha \leq d$, in accordance with (1.4). With these choices the following hold: 
(1) If $\frac{d}{2}<\kappa<\frac{d+\alpha}{2}$, then by (1.2) we obtain $V_{\kappa}(x) \asymp|x|^{-\beta}$ as in Theorem 6.1 with $\beta=\beta(\kappa)=d+\alpha-2 \kappa$. Since $0<\beta<\alpha$ for this range of $\kappa$, we are in Scenario (1) above and

$$
\varphi_{\kappa}(x) \asymp \frac{1}{(1+|x|)^{d+\alpha-\beta}},
$$

with $d+\alpha-\beta=2 \kappa$ as in Theorem 6.1 (1). Here we recover the exact asymptotic behaviour of $\varphi_{\kappa}$ at infinity.

(2) If $\kappa=\frac{d}{2}$, then by (1.2) we obtain $V_{d / 2}(x) \asymp|x|^{-\alpha} \log |x|$, and Theorem 6.2 gives that there exist $0<\gamma_{1} \leq 1 \leq \gamma_{2}$ and constants $C_{1}, C_{2}>0$ such that

$$
\frac{C_{1}}{(1+|x|)^{d}(\log (1+|x|))^{1-\gamma_{1}}} \leq \varphi_{d / 2}(x) \leq \frac{C_{2}}{(1+|x|)^{d}(\log (1+|x|))^{1-\gamma_{2}}} .
$$

Hence we are now in scenario (2) above. In particular, this means that we recover the behaviour $\varphi_{\kappa}(x) \asymp 1 /|x|^{d}$ as in (1.1), with a near miss dependent on how close $\gamma_{1} \leq 1 \leq \gamma_{2}$ are to each other. Clearly, this is a marginal and the most delicate case, and closing the gap would require a more refined analysis. Note that this special case coincides with the threshold $\kappa$ for which $\varphi_{\kappa} \notin L^{1}\left(\mathbf{R}^{d}\right)$.

(3) If $0 \vee \frac{d-\alpha}{2}<\kappa<\frac{d}{2}$, then by (1.2) we obtain $V(x) \asymp|x|^{-\alpha}$, placing this case in Scenario (3). Theorem 6.1 (2) gives that there exist $C_{1}>0$ and $\gamma=\gamma(\kappa) \in(0,1)$ such that

$$
\varphi_{\kappa}(x) \geq \frac{C_{1}}{(1+|x|)^{d-\gamma}} .
$$

Moreover, observe that for every $\kappa$ from this range and $p>\frac{d}{2 \kappa}$ it follows that $\varphi_{\kappa} \in L^{p}\left(\mathbf{R}^{d}\right)$. Then by the upper bound in Theorem 6.1 (2) we also get

$$
\varphi_{\kappa}(x) \leq \frac{C_{2}}{(1+|x|)^{2 \kappa-\varepsilon}},
$$

for every small enough $\varepsilon>0$, with some $C_{2}>0$.

When the solution of the eigenvalue equation (2.15) is antisymmetric with respect to a given hyperplane and has a definite sign in each nodal domain, then at least far away from this nodal plane some of our upper estimates improve significantly. If the solution $\varphi$ is no longer positive on $\mathbf{R}^{d}$ but it satisfies (4.10), then the upper bounds in Theorems 6.1 (1) -(2) and Theorem 6.2 (1)-(3) also hold with $\varphi$ replaced with $|\varphi|$ (this is a consequence of Theorem 4.1 (1)-(2) and Theorem 4.2 (2)). However, due to Theorem 4.2 (1), in this case the upper bound for $|\varphi|$ improves and the upper estimates in Theorems 6.1 (1) and Theorem 6.2 (1)-(2) upgrade as follows.

\section{Corollary 6.2. The following situations occur:}

(1) Under the assumptions of Theorem 6.1, if $\beta<\alpha$ and $\varphi \in C_{\mathrm{b}}\left(\mathbf{R}^{d}\right)$ is a solution of (2.15) such that (4.10) holds, then there is $C_{1}>0$ such that

$$
|\varphi(x)| \leq \frac{C_{1}}{(1+|x|)^{d+\alpha-\beta}} \frac{1}{\left(1+|x| \mathbf{1}_{\left\{\left|x_{1}\right| \geq 1\right\}}\right)^{\alpha-\beta}}, \quad x \in \mathbf{R}^{d} .
$$


(2) Under the assumptions of Theorem 6.2, if $\delta>1$ and $\varphi \in C_{\mathrm{b}}\left(\mathbf{R}^{d}\right)$ is a solution of (2.15) such that (4.10) holds, then there is $C_{2}>0$ such that

$$
|\varphi(x)| \leq \frac{C_{2}}{(1+|x|)^{d}(\log (1+|x|))^{\delta}} \frac{1}{\left(1+\mathbf{1}_{\left\{\left|x_{1}\right| \geq 1\right\}} \log |x|\right)^{\delta}}, \quad x \in \mathbf{R}^{d} .
$$

Example 6.2. Comparing these upper bounds with the exact behaviours of $\varphi_{\kappa}$ for $l=1$ and the potentials $V_{\kappa}(x) \asymp|x|^{-\beta}$ in (1.1) with $\beta=d+\alpha+2-2 \kappa, \kappa \in\left(\frac{d+2}{2}, \frac{d+\alpha+2}{2}\right)$, we see that in this case our result is not as sharp as in the cases above, however, it is still remarkably close to the exact rates. Indeed, we get here the upper bound $\left|\varphi_{\kappa}(x)\right| \leq$ $\frac{C_{1}}{(1+|x|)^{4(\kappa-1)-d}}$, while the true behaviour is $\left|\varphi_{\kappa}(x)\right| \asymp \frac{1}{|x|^{2 \kappa-1}}$. We emphasize that the symmetry/antisymmetry properties of eigenfunctions as in (4.10) are of much interest in spectral theory and are known to have important consequences (see, e.g., [26]).

Finally, we present a result for the case of fractional Schrödinger operators with potentials that are negative at infinity.

Theorem 6.3. Let $L^{(\alpha)}, 0<\alpha<2$, be a pseudo-differential operator determined by (6.1), and $V$ be an $X$-Kato class potential for which there exists $r_{0}>0$ such that $V(x) \leq 0$ and $|V(x)| \leq C|x|^{-\alpha}$, for $|x| \geq r_{0}$, with some $C>0$. Suppose that there exists a function $\varphi \in \bar{L}^{p}\left(\mathbf{R}^{d}\right)$, for some $p>1$, which is a solution to (2.15) with the property that there exists $i \in\{1,2, \ldots, d\}$ such that $\varphi\left(x_{1}, \ldots,-x_{i}, \ldots, x_{d}\right)=$ $-\varphi\left(x_{1}, \ldots, x_{i}, \ldots, x_{d}\right)$ and $\operatorname{supp} \varphi_{-} \subset\left\{x \in \mathbf{R}^{d}: x_{i} \leq 0\right\}$. Then for every $q \in\left(0, \frac{d}{p}\right)$ there exist $C=C(q)$ and $R>0$ such that

$$
|\varphi(x)| \leq C\left(\|\varphi\|_{p} \vee\|\varphi\|_{\infty}\right) \frac{1}{|x|^{q}}, \quad\left|x_{i}\right| \geq R .
$$

Proof. This is a direct application of Theorem 5.1 as now $\nu(x) \asymp|x|^{-d-\alpha}$ and $\Psi(r) \asymp$ $r^{\alpha}$.

Roughly speaking, the above result says that for potentials that are negative in a neighbourhood of infinity and for antisymmetric solutions with nodal domains in the corresponding hyperplanes, $L^{p}$-integrability, with $p>1$, always gives a polynomial decay of order near to $d / p$, far from the antisymmetry axis. This result can be compared with the examples in (1.1). Specifically, for every $i=1,2, \ldots, d$ and $l=1$ we can take $P_{i}(x)=c x_{i}$ and

$$
\varphi_{\kappa, i}(x)=\frac{c x_{i}}{\left(1+|x|^{2}\right)^{\kappa}}
$$

with $\kappa \in\left(1, \frac{d-\alpha}{2}+1\right]$. As seen in (1.3), this leads to the case of potentials $V_{\kappa, \alpha}$ negative in a neighbourhood of infinity. We clearly have $\left|\varphi_{\kappa, i}(x)\right| \leq C(1+|x|)^{-d / p}$ and $\varphi_{\kappa, i} \in$ $L^{p}\left(\mathbf{R}^{d}\right)$, for every $p>\frac{d}{2 \kappa-1}$.

6.2. Layered-type Schrödinger operators. Let $L^{(\alpha, \gamma)}, \alpha \in(0,2), \gamma>2$, be a family of self-adjoint pseudo-differential operators determined by

$$
\begin{aligned}
& L^{\widehat{(\alpha, \gamma)}} f(\xi)=-\psi^{(\alpha, \gamma)}(\xi) \widehat{f}(\xi), \quad \xi \in \mathbf{R}^{d}, \\
& f \in \operatorname{Dom}\left(L^{(\alpha)}\right)=\left\{g \in L^{2}\left(\mathbf{R}^{d}\right): \psi^{(\alpha, \gamma)} \widehat{g} \in L^{2}\left(\mathbf{R}^{d}\right)\right\},
\end{aligned}
$$


with

$$
\psi^{(\alpha, \gamma)}(\xi)=\int_{\mathbf{R}^{d} \backslash\{0\}}(1-\cos (\xi \cdot z)) v^{(\alpha, \gamma)}(z) d z
$$

where $v^{(\alpha, \gamma)}(x)=g(x /|x|)|x|^{-d-\alpha}(1 \vee|x|)^{-(\gamma-\alpha)}, d \geq 1$. Here $g: \mathbb{S}_{d} \rightarrow(0, \infty)$ is such that $g(\theta)=g(-\theta)$ and $c_{1} \leq g(\theta) \leq c_{2}$, for every $\theta \in \mathbb{S}_{d}$, with finite positive constants $c_{1}, c_{2}$. As before, every $\bar{v}^{(\alpha, \gamma)}(z) \bar{d} z$ is a symmetric Lévy measure on $\mathbf{R}^{d} \backslash\{0\}$ such that

$$
v^{(\alpha, \gamma)}(x) \asymp|x|^{-d-\alpha}(1 \vee|x|)^{-(\gamma-\alpha)}, \quad x \in \mathbf{R}^{d} \backslash\{0\} .
$$

In particular, $\int_{\mathbf{R}^{d} \backslash\{0\}} v^{(\alpha, \gamma)}(z) d z=\infty, \int_{\mathbf{R}^{d} \backslash\{0\}}|z|^{2} v^{(\alpha, \gamma)}(z) d z<\infty$, and

$$
\Psi(r) \asymp r^{2}, \quad r \in(0,1) .
$$

Moreover, it follows from [57] that the probability transition densities $p(t, x)$ exist and satisfy

$$
p(t, x) \leq c_{3}\left(\left(t^{-d / \alpha} \vee t^{-d / 2}\right) \wedge t|x|^{-d-\alpha}(1 \vee|x|)^{-(\gamma-\alpha)}\right), \quad t>0, \quad x \in \mathbf{R}^{d} .
$$

This, in particular, gives (A2) and, together with [29, Lem. 2.2], implies that Assumption (A3) holds as well. The operators $L^{(\alpha, \gamma)}$ generate the class of layered $\alpha$-stable processes.

We get the following result for potentials that are positive at infinity.

Theorem 6.4. Let $L^{(\alpha, \gamma)}, 0<\alpha<2, \gamma>2$, be a pseudo-differential operator determined by (6.6) and $V$ be an $X$-Kato class potential for which there exists $r_{0}>0$ such that $V(x)>0$ and $V(x) \asymp|x|^{-\beta}$, for $|x| \geq r_{0}$, with some $\beta>0$. Suppose that there exists a positive function $\varphi \in C_{\mathrm{b}}\left(\mathbf{R}^{d}\right)$ which is a solution of (2.15). Then the following hold:

(1) If $\beta<2$, then there exist constants $C_{1}, C_{2}>0$ such that

$$
\frac{C_{1}}{(1+|x|)^{d+\gamma-\beta}} \leq \varphi(x) \leq \frac{C_{2}}{(1+|x|)^{d+\gamma-\beta}}, \quad x \in \mathbf{R}^{d} .
$$

In particular, $\varphi \in L^{p}\left(\mathbf{R}^{d}\right)$, for every $p \geq 1$.

(2) If $\beta \geq 2$, then there exists a constant $C_{3}>0$ such that

$$
\varphi(x) \geq \frac{C_{3}}{(1+|x|)^{d+\gamma-2}}, \quad x \in \mathbf{R}^{d} .
$$

On the other hand, if $\varphi \in L^{p}\left(\mathbf{R}^{d}\right)$ for some $p>1$, then there exists $C_{4}>0$ such that

$$
\varphi(x) \leq \frac{C_{4}}{(1+|x|)^{d / p}}, \quad x \in \mathbf{R}^{d} .
$$

For potentials negative at infinity a result similar to Theorem 6.3 holds as well. 
6.3. Decay mechanisms. From the above it is seen that the decay of ground states at zero eigenvalue depends essentially on two factors. On the one hand, the sign of the potential at infinity makes a qualitative difference, and as seen in the case of classical Schrödinger operators, it has an impact even on the existence of ground states. From the decay results above one can appreciate that a positive tail of the potential has a (soft) bouncing effect tending to contain paths in compact regions, while a negative potential leaves more room for the paths to spread out to infinity. This difference makes the analysis of potentials negative at infinity much more difficult than of potentials positive at infinity.

On the other hand, the decay depends on some mean times spent in some regions by the paths. Using (2.9) and (4.17), we can give another interpretation of the results above, further highlighting the mechanisms. Assume, for simplicity, that $V(x) \asymp V^{*}(x) \asymp$ $V_{*}(x)$, for large enough $|x|$. Then we see that the conditions involving the ratios

$$
\frac{\Psi\left(\frac{1}{|x|}\right)}{V(x)} \asymp \frac{\mathbf{E}^{x}\left[\int_{0}^{\tau_{B(x,|x| / 2)}} e^{-\int_{0}^{t} V\left(X_{s}\right) d s} d t\right]}{\mathbf{E}^{0}\left[\tau_{B(0,|x|)}\right]}
$$

in Theorems 4.1-4.3 actually refer to a balance of the mean survival times of paths in a ball $B(x,|x| / 2)$ under the potential versus in the ball $B(0,|x|)$ free of the potential. Due to the doubling property, these two times are comparable, and describe specific global lifetimes (note that $B(x,|x| / 2)$ can also be replaced by $B(x, c|x|), 0<c<1$, without qualitatively changing the results). This is in sharp contrast with the case of confining potentials or decaying potentials leading to a strictly negative and sufficiently low-lying ground state eigenvalue, where the decay is governed by local lifetimes as given in (1.5). When

$$
\mathbf{E}^{x}\left[\int_{0}^{\tau_{B(x,|x| / 2)}} e^{-\int_{0}^{t} V\left(X_{s}\right) d s} d t\right]=o\left(\mathbf{E}^{0}\left[\tau_{B(0,|x|)}\right]\right)
$$

as in Scenarios (1)-(2) above, the potential has a relatively pronounced effect, making the paths favour (large) neighbourhoods of the origin than (large) neighbourhoods of far out points. This is reflected in the decay behaviours of $\varphi$ by $V$ entering explicitly in Scenario (1) discussed in Sect. 6.1. When, however,

$$
\mathbf{E}^{x}\left[\int_{0}^{\tau_{B(x,|x| / 2)}} e^{-\int_{0}^{t} V\left(X_{s}\right) d s} d t\right]=O\left(\mathbf{E}^{0}\left[\tau_{B(0,|x|)}\right]\right)
$$

as in Scenario (3), the effect of the potential is weak also in relative terms, and the two lifetimes evolve on the same scale, bordering (though clearly differing from) the situation of free fluctuations and absence of a ground state.

Acknowledgements. JL is pleased to thank Erik Skibsted for pointing out some references on the zeroeigenvalue case for classical Schrödinger operators. We also gratefully thank an anonymous Referee for a thorough reading of the manuscript and the very helpful suggestions made.

Open Access This article is distributed under the terms of the Creative Commons Attribution 4.0 International License (http://creativecommons.org/licenses/by/4.0/), which permits unrestricted use, distribution, and reproduction in any medium, provided you give appropriate credit to the original author(s) and the source, provide a link to the Creative Commons license, and indicate if changes were made.

Publisher's Note Springer Nature remains neutral with regard to jurisdictional claims in published maps and institutional affiliations. 


\section{References}

1. Agmon, S.: Lower bounds for solutions of Schrödinger equations. J. Anal. Math. 2(3), 1-25 (1970)

2. Amrein, W.O., Berthier, A.M., Georgescu, V.: Lower bounds for zero energy eigenfunctions of Schrödinger operators. Helv. Phys. Acta 57, 301-306 (1984)

3. Bardou, F., Bouchaud, J.P., Aspect, A., Cohen-Tannoudji, C.: Lévy Statistics and Laser Cooling. Cambridge University Press, Cambridge (2003)

4. Benguria, R., Yarur, C.: Sharp condition on the decay of the potential for the absence of a zero-energy ground state of the Schrödinger equation. J. Phys. A 23, 1513-1518 (1990)

5. Bertolotti, J., et al.: Engineering disorder in superdiffusive Lévy glasses. Adv. Funct. Mater. 20, 965-968 (2010)

6. Bogdan, K., Kumagai, T., Kwaśnicki, M.: Boundary Harnack inequality for Markov processes with jumps. Trans. Am. Math. Soc. 367, 477-517 (2015)

7. Boretz, Y., et al.: Optically tunable bound states in the continuum. Phys. Rev. A 90, 023853 (2014)

8. Capasso, F., et al.: Observation of an electronic bound state above a potential well. Nature 358, 565-567 (1992)

9. Christ, M., Kiselev, A.: One-dimensional Schrödinger operators with slowloy decaying potentials: spectra and asymptotics, notes to Workshop on Oscillatory Integrals and Dispersive Equations, IPAM (2001)

10. Cuenin, J.-C.: Embedded eigenvalues of generalized Schrödinger operators. J. Spectr. Theory (to appear)

11. Demuth, M., van Casteren, J.A.: Stochastic Spectral Theory for Selfadjoint Feller Operators. Probability and its Applications. Birkhäuser Verlag, Basel, A Functional Analysis Approach (2000)

12. Denisov, S., Kiselev, A.: Spectral properties of Schrödinger operators with decaying potentials. In: Proceedings of Symposia in Pure Mathematics (B. Simon Festschrift), vol. 76. AMS (2007)

13. Dereziński, J., Skibsted, E.: Quantum scattering at low energies. J. Funct. Anal. 257, 1828-1920 (2009)

14. Dong, J.: Scattering problems in the fractional quantum mechanics governed by the $2 D$ space-fractional Schrödinger equation. J. Math. Phys. 55, 032102 (2014)

15. Durugo, S.O., Lórinczi, J.: Spectral properties of the massless relativistic quartic oscillator. J. Differ. Equ. 264, 3775-3809 (2018)

16. Eastham, M.S.P., Kalf, H.: Schrödinger-Type Operators with Continuous Spectra. Pitman, London (1982)

17. Fall, M.M., Felli, V.: Unique continuation property and local asymptotics of solutions to fractional elliptic equations. Commun. Partial Differ. Equ. 39, 354-397 (2014)

18. Fournais, S., Skibsted, E.: Zero energy asymptotics of the resolvent for a class of slowly decaying potentials. Math. Z. 248, 593-633 (2004)

19. Głowacki, P., Hebisch, W.: Pointwise estimates for densities of stable semigroups of measures. Studia Math. 104, 243-258 (1993)

20. Herbst, I., Skibsted, E.: Decay of eigenfunctions of elliptic PDE's. I. Adv. Math. 270, 138-180 (2015)

21. Hinz, A.M.: Obere Schranken für Eigenfunktionen eines Operators $-\Delta+q$. Math. Z. 185, 291-304 (1984)

22. Ikeda, N., Watanabe, S.: On some relations between the harmonic measure and the Lévy measure for a certain class of Markov processes. J. Math. Kyoto Univ. 2-1, 79-95 (1961)

23. Jacob, N.: Pseudo-Differential Operators and Markov Processes, vols. 1-3. Imperial College Press, London (2003-2005)

24. Jäh, C., Lőrinczi, J.: Eigenvalues at the continuum edge for fractional Schrödinger operators, preprint (2019)

25. Jensen, A., Kato, T.: Spectral properties of Schrödinger operators and time-decay of the wave functions. Duke Math. J. 46, 583-611 (1979)

26. Kaleta, K.: Spectral gap lower bound for the one-dimensional fractional Schrödinger operator in the interval. Studia Math. 209, 267-287 (2012)

27. Kaleta, K., Lőrinczi, J.: Pointwise estimates of the eigenfunctions and intrinsic ultracontractivity-type properties of Feynman-Kac semigroups for a class of Lévy processes. Ann. Probab. 43, 1350-1398 (2015)

28. Kaleta, K., Lőrinczi, J.: Transition in the decay rates of stationary distributions of Lévy motion in an energy landscape. Phys. Rev. E 93, 022135 (2016)

29. Kaleta, K., Lőrinczi, J.: Fall-off of eigenfunctions for non-local Schrödinger operators with decaying potentials. Potential Anal. 46, 647-688 (2017)

30. Kenig, C.E.: Restriction theorems, Carleman estimates, uniform Sobolev inequalities and unique continuation. In: Harmonic Analysis and Partial Differential Equations (El Escorial, 1987). In: García-Cuerva J. (ed.) Harmonic Analysis and Partial Differential Equations. Lecture Notes in Mathematics, vol. 1384, pp. 69-90. Springer (1989)

31. Kenig, C.E., Nadirashvili, N.: A counterexample in unique continuation. Math. Res. Lett. 7, 625-630 (2000)

32. Knowles, I.: On the number of $L^{2}$-solutions of second order linear differential equations. Proc. R. Soc. Edinb. Sect. A 80, 1-13 (1978) 
33. Knowles, I.: On the location of eigenvalues of second-order linear differential operators. Proc. R. Soc. Edinb. Sect. A 80, 15-22 (1978)

34. Koch, H., Tătaru, D.: Sharp counterexamples in unique continuation for second order elliptic equations. J. Reine Angew. Math. 542, 133-146 (2002)

35. Lieb, E.: Thomas-Fermi and related theories of atoms and molecules. Rev. Mod. Phys. 53, 603-641 (1981)

36. Longhi, S.: Fractional Schrödinger equation in optics. Opt. Lett. 40, 1117-1120 (2015)

37. Lőrinczi, J., Hiroshima, F., Betz, V.: Feynman-Kac-Type Theorems and Gibbs Measures on Path Space. With Applications to Rigorous Quantum Field Theory, de Gruyter Studies in Mathematics, vol. 34, Walter de Gruyter (2011), 2nd edn (2019)

38. Lőrinczi, J., Małecki, J.: Spectral properties of the massless relativistic harmonic oscillator. J. Differ. Equ. 253, 2846-2871 (2012)

39. Lőrinczi, J., Sasaki, I.: Embedded eigenvalues and Neumann-Wigner potentials for relativistic Schrödinger operators. J. Funct. Anal. 273, 1548-1575 (2017)

40. Lőrinczi, J., Sasaki, I.: Absence of embedded eigenvalues for a class of non-local Schrödinger operators, preprint (2019)

41. Maceda, M.: On the Birman-Schwinger principle applied to $\sqrt{-\Delta+m^{2}}-m$. J. Math. Phys. 47, 033506 (2006)

42. Marinica, D.C., Borisov, A.G., Shabanov, S.V.: Bound states in the continuum in photonics. Phys. Rev. Lett. 100, 183902 (2008)

43. Nakamura, S.: Low-energy asymptotics for Schrödinger operators with slowly decreasing potentials. Commun. Math. Phys. 161, 63-76 (1994)

44. Newton, R.G.: Nonlocal interactions: the generalized Levinson theorem and the structure of the spectrum. J. Math. Phys. 18(1348-1357), 1582-1588 (1977)

45. Pruitt, W.E.: The growth of random walks and Lévy processes. Ann. Probab. 9, 948-956 (1981)

46. Ramm, A.G.: Sufficient conditions for zero not to be an eigenvalue of the Schrödinger operator. J. Math. Phys. 28, 1341-1343 (1987)

47. Ramm, A.G.: Sufficient conditions for zero not to be an eigenvalue of the Schrödinger operator. J. Math. Phys. 29, 1431-1432 (1988)

48. Reed, M., Simon, B.: Methods of Modern Mathematical Physics, vol. 3-4. Academic Press, London (1979)

49. Richard, S., Umeda, T.: Low energy spectral and scattering theory for relativistic Schrödinger operators. Hokkaido Math. J. 45, 141-179 (2016)

50. Rüland, A.: Unique continuation for fractional Schrödinger equations with rough potentials. Commun. Partial Differ. Equ. 40, 77-114 (2015)

51. Sato, K.I.: Lévy Processes and Infinitely Divisible Distributions. Cambridge University Press, Cambridge (1999)

52. Schilling, R.: Growth and Hölder conditions for the sample paths of Feller processes. Probab. Theory Relat. Fields 112, 565-611 (1998)

53. Seo, I.: On unique continuation for Schrödinger operators of fractional and higher orders. Math. Nachr. 287, 699-703 (2014)

54. Seo, I.: Unique continuation for fractional Schrödinger operators in three and higher dimensions. Proc. AMS 143, 1661-1664 (2015)

55. Skibsted, E., Wang, X.P.: Two-body threshold spectral analysis, the critical case. J. Funct. Anal. 260, 1766-1794 (2011)

56. Simon, B.: Large time behavior of the $L^{p}$ norm of Schrödinger semigroups. J. Funct. Anal. 40, 66-83 (1981)

57. Sztonyk, P.: Estimates of tempered stable densities. J. Theor. Probab. 23, 127-147 (2010)

58. von Neumann, J., Wigner, E.: Über merkwürdige diskrete Eigenwerte. Z. Phys. 30, 465-467 (1929)

59. Yafaev, D.: The low energy scattering for slowly decreasing potentials. Commun. Math. Phys. 85, 177-196 (1982)

60. Zhang, X., et al.: The transition of energy and bound states in the continuum of fractional Schrödinger equation in gravitational field and the effect of the minimal length. Commun. Nonlinear Sci. Numer. Simul. 67, 290-302 (2019)

61. Zhang, Y., et al.: Propagation dynamics of a light beam in a fractional Schrödinger equation. Phys. Rev. Lett. 115, 180403 (2015)

62. Zhang, Y., et al.: Resonant mode conversions and Rabi oscillations in a fractional Schrödinger equation. Opt. Express 25, 32401-32410 (2017) 